\title{
Comparison of eight years magnetic field data from Cluster with Tsyganenko models in the inner magnetosphere
}

\author{
Q.-H. Zhang ${ }^{1,2, *}$, M. W. Dunlop ${ }^{2}$, R. Holme ${ }^{1}$, and E. E. Woodfield ${ }^{3}$ \\ ${ }^{1}$ Department of Earth and Ocean Sciences, University of Liverpool, Liverpool, UK \\ ${ }^{2}$ Space Science and Technology Department, Rutherford Appleton Laboratory, Chilton, Didcot, Oxfordshire, UK \\ ${ }^{3}$ Department of Communications Systems, University of Lancaster, Lancaster, UK \\ *now at: SOA Key Laboratory for Polar Science, Polar Research Institute of China, Shanghai, 200136, China
}

Received: 26 August 2009 - Revised: 8 January 2010 - Accepted: 14 January 2010 - Published: 29 January 2010

\begin{abstract}
Eight years of magnetic field data, taken while the four Cluster spacecraft pass through, or adjacent to, the equatorial ring current, have been surveyed to investigate the effects on the Earth's magnetic field of the externally driven current systems connecting the ionosphere, cusp and ring current regions. This study extends previous work to cover a greater range of orbit location and external conditions. We compare the modeled magnetic field from different global field models (Tsyganenko, 1989, 1996, and 2001, hereafter T89, T96 and T01) with data from the four Cluster spacecraft. Comparing with the different models allow us not only to characterize each model's performance, but also provides insight into the physical sources of observed signals. The data generally deviate much less from the expected model field during the years close to the solar minimum, implying that the models perform better during weaker geomagnetic activity. There are particular deviations from the models associated with the ring current (well-defined smooth trends) and region 2 field aligned currents (FACs) or low-altitude cusp FACs (sharp bipolar signatures). During the ring current crossings (through perigee, at $4-5 R_{\mathrm{E}}$ ), the T96 model always overestimates the ring current, while the T01 and T89 models sometimes underestimate it. The sharp bipolar signatures are not always sampled, implying a localized extent, but only the T96 and T01 models include forms for the region 2 FACs and T01 appears to model these better. Overall, all deviations from T01 are much smaller than for the other models, indicating that this model achieves the best fit to the data. The 4 Cluster spacecraft observe nearly the same signatures at small separations (during the early years of the mission) but do sample different signatures at the large separations (during the later years). Using the four-spacecraft technique, we infer that the region 2 FACs, with a transverse
\end{abstract}

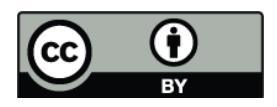

Correspondence to: Q.-H. Zhang (qinghe.zhang@stfc.ac.uk) thickness of $\sim 0.17-0.54 R_{\mathrm{E}}$, and cusp FACs, with a thickness of $\sim 0.06-0.12 R_{\mathrm{E}}$, are very stable in size and location.

Keywords. Magnetospheric physics (Current systems; Magnetopause, cusp, and boundary layers; Magnetospheric configuration and dynamics)

\section{Introduction}

Woodfield et al. (2007) surveyed the contribution of ionospheric and magnetospheric current systems to the Earth's magnetic field, by using perigee pass data from the Cluster spacecraft, based on predictions of the Tsyganenko 2001 (T01) (Tsyganenko, 2002a, b) global field model. The results showed that the T01 model performs very well in a global sense, although absolute residuals between the data and the model can reach $\sim 20 \mathrm{nT}$ near perigee, often with stable bipolar signatures, which repeat on the phase period of the Cluster orbit and were assumed to be observed field-aligned currents (FACs). In order to further investigate the external current systems in the magnetosphere, this paper extends the study of Woodfield et al. (2007) to compare eight years magnetic field data from the 4-spacecraft Cluster array with the Tsyganenko models.

The semi-empirical Tsyganenko magnetic field models have been widely utilized in the space physics community for many years. They are best-fit representations for the magnetic field, based on a large number of satellite observations (IMP, HEOS, ISEE, POLAR, Geotail, GOES, etc). The models include contributions from major external magnetospheric sources: the ring current, magnetotail current system, magnetopause currents, and the large-scale system of fieldaligned currents.

There are currently four main versions. The 1989 version (T89) (Tsyganenko, 1989) was primarily developed as

Published by Copernicus Publications on behalf of the European Geosciences Union. 
a simple empirical approximation for the global magnetosphere, binned into several intervals of the disturbance index Kp. The 1996 version (T96) (Tsyganenko, 1996) has an explicitly defined realistic magnetopause, large-scale Region 1 and 2 Birkeland current systems, and Interplanetary Magnetic Field (IMF) penetration across the boundary. T96 is parameterized by the solar wind ram pressure, disturbance storm time index (Dst (Sugiura and Kamei, 1991) or its high time resolution counterpart SYM-H (Wanliss and Showalter, 2006)), and transverse components ( $B_{\mathrm{Y}}$ and $\left.B_{\mathrm{Z}}\right)$ of the IMF. The 2001 version (T01) (Tsyganenko, 2002a, b) represents the variable configuration of the inner and near magnetosphere for different interplanetary conditions and ground disturbance levels. It also takes into account the observed dawn-dusk asymmetry of the inner magnetosphere due to the partial ring current that develops during magnetospheric disturbances. The T01 model is driven by the same input parameters as the T96 model: solar wind ram pressure, Dst or SYM-H, and transverse components $\left(B_{Y}\right.$ and $\left.B_{\mathrm{Z}}\right)$ of the IMF, but it also requires a one-hour time history of external inputs to the magnetosphere. The 2004 version (T04S) (Tsyganenko, 2005) is a dynamical model of the storm-time geomagnetic field in the inner magnetosphere, based on space magnetometer data taken during 37 major events in 1996-2000 and concurrent observations of the solar wind and IMF. Most of the data set used in the current study does not correspond to storm time conditions, so only the first three model versions have been used here.

In order to analyze and characterize the modelled and actual magnetospheric field contributions, the output from the T89, T96 and T01 models has been compared to magnetic field data from the Cluster mission (Escoubet et al., 2001) during its ring current crossing (through perigee, at 4-5 $R_{\mathrm{E}}$ ). Orbits separated in time but "in-phase" relative to the dayside, nightside and dawn-dusk flanks are compared, such that the changes in ring current and FACs existing in the magnetosphere are investigated. The results from a particular orbit sample are shown to recur in an analysis of eight full years of data. Such a comparison with Cluster data provides an independent assessment of the accuracy of the Tsyganenko models, since these data are not included in the empirical database of the models. This comparison also allows the magnetic field behaviour observed by Cluster to be assessed in a large-scale context.

\section{Data sources and methodology}

\subsection{Field models}

Since the Tsyganenko models represent only the magnetospheric contributions to the overall magnetic field of the Earth, we have used the International Geomagnetic Reference Field (IGRF) version 10 (Maus et al., 2005a, b) to represent the Earth's internal magnetic field in order to model the full magnetic field at the Cluster orbit. The following analysis assumes that both the ionospheric and crustal contributions to the magnetic field at the altitude of the Cluster orbit are not significant.

The mathematical structure of the T89 model includes the major magnetospheric current systems: magnetotail current system, ring current, and Chapman-Ferraro currents, while the T96 model adds the large-scale Region 1 and 2 Birkeland current systems and a parameterisation for the interplanetary magnetic field penetration. In addition to the current systems mentioned above, the T01 model also includes the partial ring current with the associated dawn-dusk asymmetry of the inner magnetosphere, and a more detailed form of partial IMF penetration. The pertinent features of these current structures are summarized below. For a full description the reader is referred to Tsyganenko $(1989,1996,2002 a, b)$ and references therein.

The T01 ring current includes both an axisymmetric and a partial ring current, with field-aligned closure currents. The cross-tail current sheet, which is allowed to warp in response to the geo-dipole tilt, has a thickness that varies both across and along the tail. The location of the inner boundary of this current sheet along the Sun-Earth line varies with changing geomagnetic disturbance levels. The contribution of the magnetopause currents to the total magnetic field is represented using a potential field and is optimized so that, when added to the field from internal sources, it provides the required distribution of the net normal component at the model magnetopause boundary. The general magnetopause shape is defined by the empirical model of Shue et al. (1998) and is also allowed to be geo-dipole tilt-dependent. The FAC representation includes both region 1 and region 2 FACs (Iijima and Potemra, 1976) which are allowed to vary with interplanetary conditions such that their ionospheric footprints can move in latitude. Finally, the interconnection field, controlled by the IMF, allows the model magnetosphere to assume open configurations (by allowing a finite $B$ normal to the magnetopause).

The magnitude of the total external magnetic field contributions from the Tsyganenko models can reach tens of nanoTesla within $\pm 1 \mathrm{~h}$ of Cluster perigee. The majority of this is due to the ring current since, at perigee, Cluster is close to $4 R_{\mathrm{E}}$ radial distance and passes adjacent to or through the ring current region. At that distance, the dominant IGRF contribution from the Earth's internal magnetic field is hundreds of nano-Tesla.

The other field models use similar mathematical structure, but with different emphasis and definitions of the various sources. For the intervals covered in this paper, the geomagnetic activity input to the T89 model is provided by the $\mathrm{Kp}$ index resulting in quasi-static model output. The interplanetary input data for the T96 and T01 models (i.e. solar wind dynamic pressure, IMF $B_{\mathrm{Y}}$ and $B_{\mathrm{Z}}$ ) were the lagged solar wind and magnetic field data from the OMNIWeb site (http://omniweb.gsfc.nasa.gov/); an extra shift of $5 \mathrm{~min}$ has 

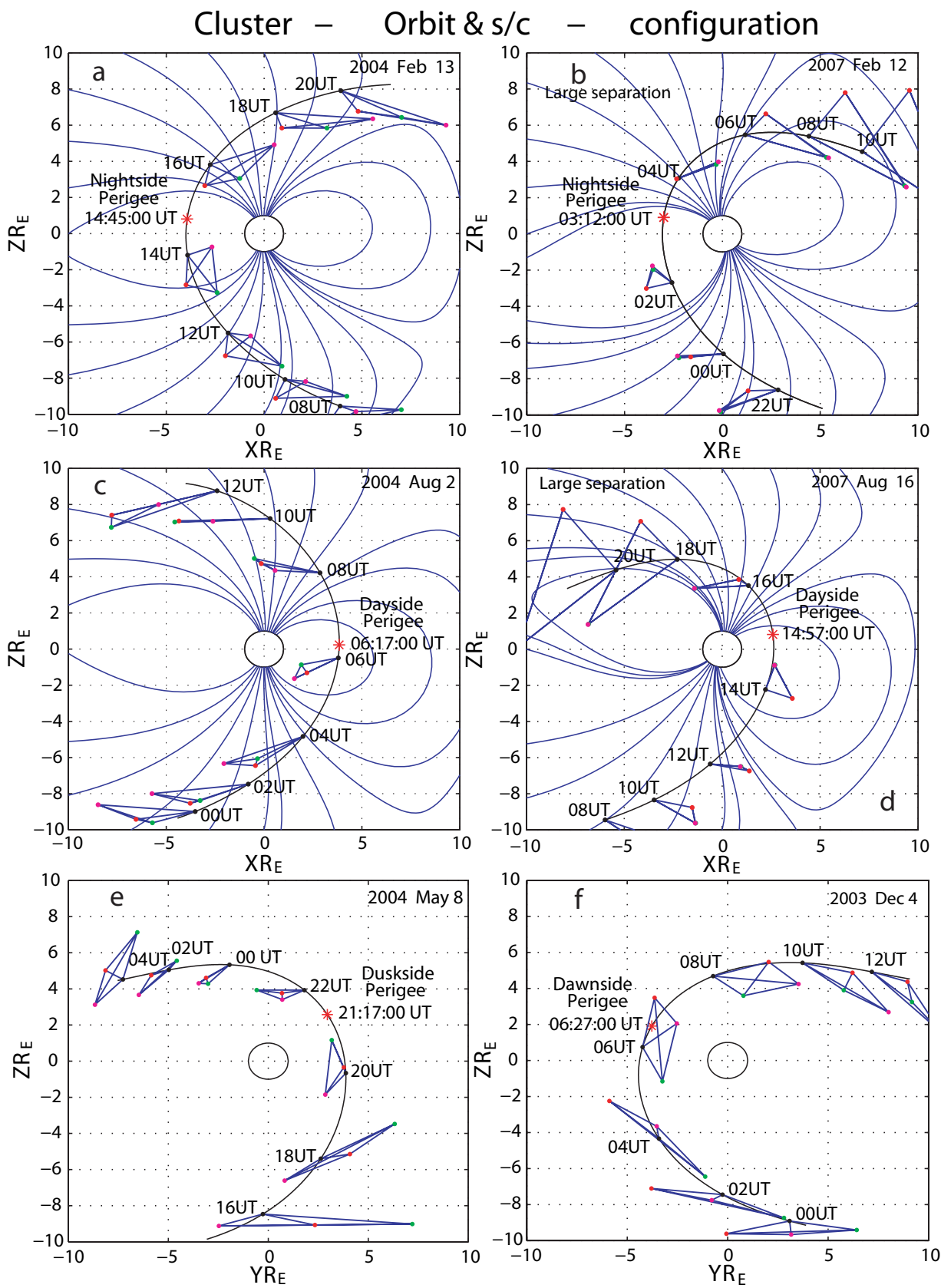

Fig. 1. Orbit plots in XZ or YZ plane in GSM coordinates for night-side orientations orbit 558/559 on 13 February 2004 (a) and 1018/1019 on 12 February 2007 (b), for dayside orientations orbit 630/631 on 2 August 2004 (c) and 1096/1097 on 16 August 2007 (d), and for duskdawn orientation orbit 594/595 on 8 May 2004 (e, duskside) and 528/529 on 4 December 2003 (f, dawnside), respectively. The orbit also shows the configuration of the Cluster spacecraft array as a tetrahedron (size scaled up by a factor of 80 for Fig. 1a and f, 5 for Fig. $1 \mathrm{~b}$ and d, and 20 for Fig. 1c and e). Model geomagnetic field lines are drawn from the T01 model with the average inputting parameters during the interval of interest on each orbit.

been added to the OMNIWeb data to account for the convection from the bow shock nose to magnetopause. A one-hour time history of solar wind data was used to calculate the parameters $g_{1}$ and $g_{2}$ for T01 (Tsyganenko, 2002b). The geomagnetic activity input to T96 and T01 is provided by the dynamic SYM-H index (Wanliss and Showalter, 2006).

\subsection{Cluster data}

The ESA Cluster mission is composed of an array of four spacecraft carrying identical payloads. The spacecraft were launched in pairs in July and August 2000 into similar elliptical, polar orbits, each with a perigee of $\sim 4 R_{\mathrm{E}}$, an apogee 
Table 1. Input parameters for T01 model as used in Fig. 1.

\begin{tabular}{lcccc}
\hline Figure & $\begin{array}{c}\text { Pdyn } \\
(\mathrm{nPa})\end{array}$ & $\begin{array}{c}\text { IMF } B_{\mathrm{Y}} \\
(\mathrm{nT})\end{array}$ & $\begin{array}{c}\text { IMF } B_{\mathrm{Z}} \\
(\mathrm{nT})\end{array}$ & $\begin{array}{c}\text { Dst } \\
(\mathrm{nT})\end{array}$ \\
\hline Fig. 1a & 1.66 & -2.38 & -4.55 & -25 \\
Fig. 1b & 1.16 & -3.75 & -0.57 & -9 \\
Fig. 1c & 0.71 & -1.24 & -0.62 & -14 \\
Fig. 1d & 1.36 & -4.10 & -0.62 & -10 \\
Fig. 1e & 0.93 & -3.32 & 1.42 & -27 \\
Fig. 1f & 1.05 & -3.11 & -3.87 & -1 \\
\hline
\end{tabular}

of $\sim 19.6 R_{\mathrm{E}}$ and identical orbital periods of $57 \mathrm{~h}$. A typical orbital orientation with respect to the model field lines is shown in Fig. 1. Due to the Earth's orbital motion, Cluster's orbit precesses in the Geocentric Solar Magnetospheric (GSM) coordinate system, so that every year all magnetic local times (MLT) are covered. The orbital parameters of each spacecraft produce a tetrahedral spacecraft formation, which evolves around the orbit. The orbits are adjusted approximately once every 6 months via a sequence of manoeuvres to vary the spatial scales between $100 \mathrm{~km}$ and a few $R_{\mathrm{E}}$. Each Cluster spacecraft has eleven experiments on board; here, we have used $1 \mathrm{~min}$ averaged data from the fluxgatemagnetometer (FGM) (Balogh et al., 2001). In-flight calibrations on the FGM data routinely determine the maximum error in the data for each spacecraft to within $0.1 \mathrm{nT}$.

\subsection{Method}

We have used a combination of approaches to compare the residuals between different models and between different spacecraft to reveal pertinent features in the data. Firstly, we have compared the predicted contributions to the magnetic field from the various external current systems at the orbit tracks of Cluster, using the outputs from T89, T96 and T01 models, respectively, to assess the behaviour of the different models. Secondly, we have analysed the magnetic field data observed by Cluster S/C 1 by differencing (subtracting) the predicted static IGRF $\left(\boldsymbol{B}_{\mathrm{EO}}=\boldsymbol{B}_{\text {Observed }}-\boldsymbol{B}_{\mathrm{IGRF}}\right)$ and the modeled magnetic field data $\left(d \boldsymbol{B}=B_{\text {Observed }}-\boldsymbol{B}_{-T}-\boldsymbol{B}_{\mathrm{IGRF}}\right.$, where $\boldsymbol{B}_{-\mathrm{T}}$ is the Tsyganenko magnetic field) from T89, T96, and T01, respectively. Finally, we have compared the magnetic field data from the 4 Cluster spacecraft with that modeled by T01 to make spatial comparisons during similar external conditions. This methodology is designed to unravel the predicted and measured effects of both the time dependence and spatial sampling of the external current systems.

In the following assessment, the data residuals are formed by subtracting the model values of magnetic field (the Tsyganenko model and/or IGRF) from the data (note that we have used absolute rather than percentage residuals). A positive residual, therefore, suggests the model value is too small.
GSM coordinates are used throughout. The Cluster orbits are, by convention, numbered from perigee to perigee and, for our purpose to generate a set of data for the whole available eight years of the Cluster mission, we have investigated partial orbits centered on perigee. Data from all 4 Cluster spacecraft are used for these comparisons.

\section{The modeled and measured external current contributions}

Figure 1a and $\mathrm{b}$ shows two orbit plots projected into the $\mathrm{XZ}$ plane in GSM coordinates for orbit 558/559 on 13 February 2004 and 1018/1019 on 12 February 2007, which represent two night-side orientations at small and large spacecraft separations. Figure 1c and d shows orbits 630/631 on 2 August 2004 and 1096/1097 on 16 August 2007, which represent the dayside configurations at small and large separations, passing through the cusp. Figure 1e and $\mathrm{f}$ shows the $\mathrm{YZ}$ plane in GSM for orbits 594/595 on 8 May 2004 and 528/529 on 4 December 2003, representing the dawn-dusk, flank orientations. The plots show the configuration of the Cluster spacecraft array as a tetrahedron every two hours along the orbit (size scaled up by a factor of 80 for Fig. 1a and f, 5 for Fig. 1b and d, and 20 for Fig. 1c and e). Model geomagnetic field lines are drawn from the T01 model with the input parameters given in Table 1.

\subsection{Cluster orbit coverage}

From Fig. 1a (b), we find that on 13 February 2004 (12 February 2007) the spacecraft moved from the pre-midnight (midnight) sector south of the magnetic equator through perigee at 01:00 MLT (02:00 MLT) to the midnight (premidnight) sector north of the equator. The Cluster spacecraft array passed through or near the outer radiation belt or ring current near to perigee and passed into the high-latitude cusp region in the Northern Hemisphere after about 19:00 UT on 13 February 2004 and after 06:00 UT on 12 February 2007. Comparing Fig. 1a and b, we find the Cluster orbit has clearly dropped southward by 12 February 2007, which leads to a middle altitude cusp crossing about $2 \mathrm{~h}$ earlier than that on 13 February 2004, and demonstrates the southward motion of the Cluster apogee in later years. For the dayside perigees (Fig. 1c and d), the change in the line of apsides is not so significant. From Fig. 1c (d), we find that on 2 August 2004 (16 August 2007) the spacecraft moved from the postnoon sector south of the magnetic equator through perigee at 14:00 MLT to the pre-noon sector north of the equator. The Cluster spacecraft array passed through or near the ring current close to perigee and passed into the low altitude cusp region in the Northern Hemisphere after about 08:00 UT on 2 August 2004 and after 15:00 UT on 16 August 2007. The comparison from Fig. $1 \mathrm{~b}$ and e also shows the southward motion of the Cluster apogee in later years. In addition, the 

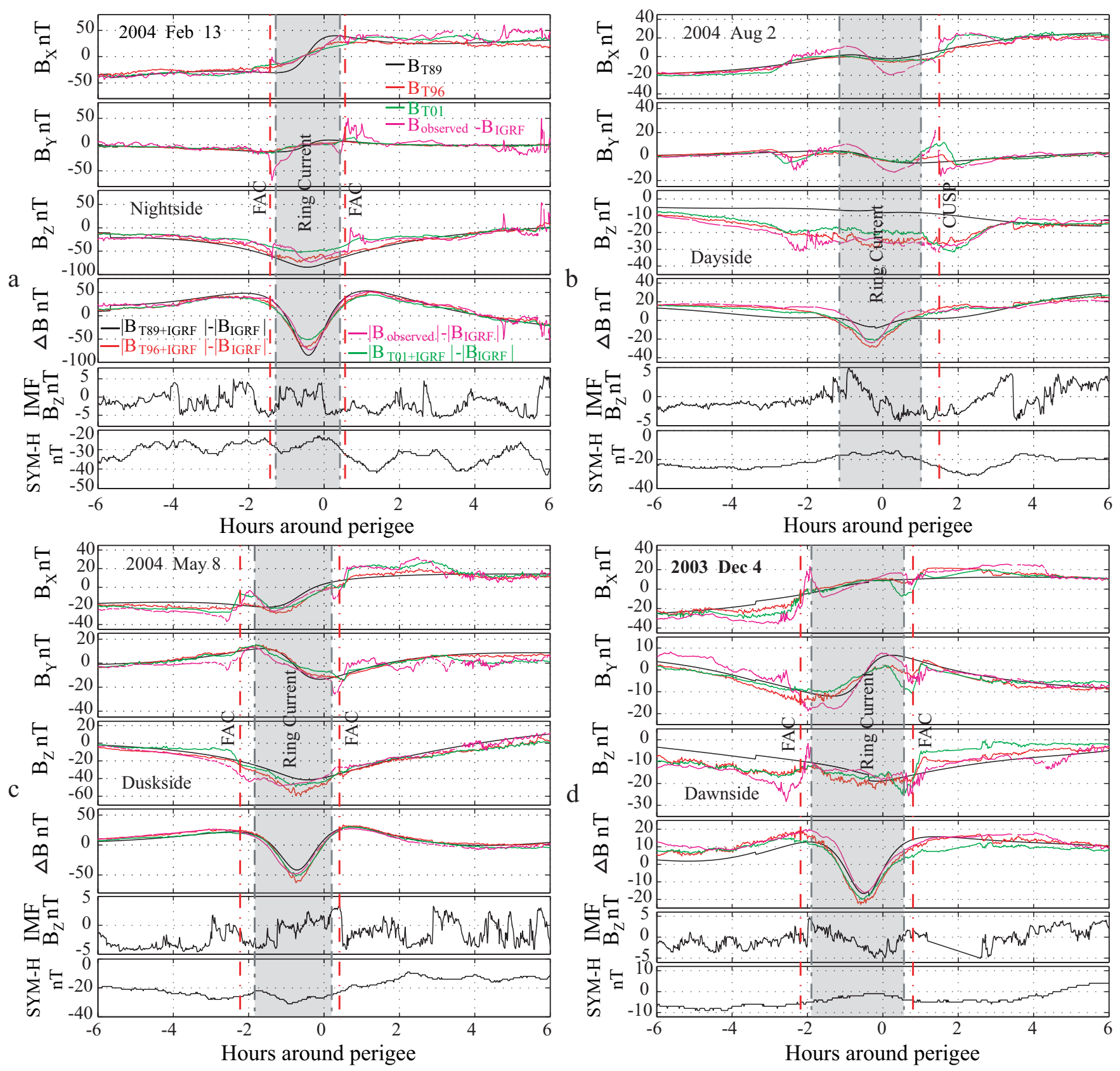

Fig. 2. External magnetic field predicted by T89 (black), T96 (red) and T01 (green) models and measured by Cluster S/C 1 (the observations minus the predictions from IGRF model, magenta) at the tracks of Cluster S/C 1 on 13 February 2004 (a), 2 August 2004 (b), 8 May 2004 (c) and 4 December 2003 (d), respectively, together with the IMF $B_{Z}$ and SYM-H index. From top to bottom panels in each figure are for the $X_{\mathrm{GSM}}, Y_{\mathrm{GSM}}, Z_{\mathrm{GSM}}$ and the magnitude of the field. The black vertical dashed lines show the ring current region crossing.

Cluster array shows that Cluster S/C 3 and 4 move close together in the orbit phase for the 12 February and 16 August 2007, which leads to triangular rather than tetrahedral configurations. The dawn-dusk flank orbits show the spacecraft moves from dawnside (duskside) sector south of the magnetic equator through perigee at 20:30 MLT (07:00 MLT) on the duskside (dawnside) and back to the dawnside (dusk- side) sector north of the equator on 8 May 2004 (4 December 2003).

Since the orbital period is $57 \mathrm{~h}$, successive passes do not cross perigee at the same time of day, i.e. for the same dipole tilt. This issue has already been discussed by Woodfield et al. (2007), who chose to compare every 8th orbit in pairs (19 days apart) in order to sample the same dipole phase. In this 
paper we acknowledge the issue of changing magnetospheric locations, using the Bryant style format (Bryant et al., 1985) to summarise the magnetic sampling during the mission, and concentrate on the comparative modelled and actual residuals monitored over the wider data range (these plots are 2-D colour plots with the colour scale representing the residuals, the $\mathrm{x}$-axis representing the orbit number and $\mathrm{y}$-axis being time relative to perigee).

\subsection{Comparison of the output from different Tsyga- nenko models and Cluster S/C 1}

In Fig. 2, we show the predicted external magnetic field contributions from the T89 (black), T96 (red) and T01 (green) models for the MLT sectors: nightside, dayside, duskside and dawnside. The estimated external field contributions observed by Cluster S/C1 $\left(\boldsymbol{B}_{\mathrm{EO}}=\boldsymbol{B}_{\text {Observed }}-\boldsymbol{B}_{\mathrm{IGRF}}\right)$ at the tracks of Cluster S/C 1 is shown in magenta. From top to bottom, the panels are the $B_{\mathrm{XGSM}}, B_{\mathrm{YGSM}}, B_{\mathrm{ZGSM}}$, the magnitude of the field, IMF $B_{\mathrm{Z}}$ and SYM-H index.

Figure 2a shows the night-side pass from 13 February 2004. During the interval of interest, the predictions of all three models (for example, of the ring current field) are similar. The approximate times of entry into and exit from the ring current region of Cluster S/C 1 are highlighted by the grey region between the two grey vertical dashed lines (this was determined by the high electron and proton flux observed by RAPID (Wilken et al., 2001), not shown here). From Fig. 2a, we find that the predicted components from the three models vary as follows: the $B_{\mathrm{X}}$ components vary from negative (about $-40 \mathrm{nT}$ ), via zero in the ring current region, to positive (about $+40 \mathrm{nT}$ ) (see first panel in Fig. 2a); the $B_{Y}$ components are near zero at the beginning and the end of this interval, and vary from negative (about $-15 \mathrm{nT}$ ), via zero at the middle of the ring current, to positive (about $+10 \mathrm{nT}$ ) (see second panel in Fig. 2a); the $B_{\mathrm{Z}}$ components are almost always negative and decrease during pre-perigee crossing to reach about $-50 \sim-80 \mathrm{nT}$ (i.e. for T01, $\sim-50 \mathrm{nT}$ , for T96, $\sim-70 \mathrm{nT}$, for T89, $\sim-80 \mathrm{nT}$ ) at the middle of ring current region (see third panel in Fig. 2a). $\Delta B_{\text {model }}$ $\left(\Delta B_{\text {model }}=\left|B_{\mathrm{IGRF}}+B_{\text {model }}\right|-\left|B_{\mathrm{IGRF}}\right|\right)$ shows an "M" shape with minimum magnitude of about $-50 \sim-80 \mathrm{nT}$ in this region (i.e. for T01, $\sim-50 \mathrm{nT}$, for T96, $\sim-65 \mathrm{nT}$, for T89, $\sim-80 \mathrm{nT}$ ) (see fourth panel in Fig. 2a). This signature arises because the combined field of the ring and tail currents is directed southward, opposite to the main field, in the equatorial inner magnetosphere (Sugiura et al., 1971).

For the February 2004 pass, the magnitude estimated by T89 is maximum at the center of the ring current crossing, and shows the most significant deviation in the $B_{\mathrm{X}}$ and $B_{\mathrm{Z}}$ components, overestimating the observed ring current. In contrast T96 produced the biggest magnitude at the center of ring current crossing for most of the eight years data, as is shown in some detail below. The magnetic field estimated by the quasi-static T89 model (input of a single $\mathrm{Kp}$ at $3 \mathrm{~h}$ resolution) shows the smoothest features with a small jump at the beginning when the Kp index changed (the Kp index changes to $4+$ at the beginning and remains at $4-$ for the later interval). The dynamic output from the T96 and T01 models (through input of solar wind dynamic pressure, IMF $B_{Y}$ and $B_{\mathrm{Z}}$, and SYM-H at 1 min resolution) in Fig. 2 shows that they predict the magnetic field in the region $2 \mathrm{FAC}$ at both boundaries of the ring current, but at a lower amplitude than observed. This is especially clear in the $B_{\mathrm{Y}}$ component, where T01 estimated a bigger signature than T96. In fact, the observed field excursions are more than twice those of the T01 model. During this interval, the solar wind dynamic pressure varied between $\sim 1.2-2.8 \mathrm{nPa}$, IMF $B_{\mathrm{Z}}$ and $B_{\mathrm{Y}}$ in GSM varied between -5 and $+5 \mathrm{nT}$, and SYM-H varied between -42 and $-22 \mathrm{nT}$ (see the bottom two panels in Fig. 2a).

Figure $2 b$ shows the dayside pass from 2 August 2004 where the low-altitude cusp is encountered (see Fig. 1c). This figure shows the $B_{\mathrm{Y}}$ component predicted by T01 (green line) is much larger than that from the other models near the boundary of the ring current, whereas the Cluster observations (magenta line) show a persistent ring current signature not predicted by the models. During this pass, the IMF $B_{Z}$ varies between about -5 and $5 \mathrm{nT}$, and $\mathrm{SYM}-\mathrm{H}$ varies between about -15 and $-30 \mathrm{nT}$ through the central region. The $B_{\mathrm{X}}$ components from the three models increase from negative (about $-20 \mathrm{nT}$ ) to positive near the boundary of the ring current region, slightly decrease to negative (about $-5 \mathrm{nT}$ ) at the center of the ring current region and then increase (crossing zero near the boundary of the ring current region) again to positive (about $+20 \mathrm{nT}$ ) (see first panel in Fig. 2b). The $B_{X}$ and $B_{Y}$ components in the measured data are near zero at the beginning and the end of this interval and vary like a distorted "S" shape, which slightly increases to positive (about $8 \mathrm{nT}$ ), then decreases to negative in the ring current region (later increasing to near zero about $2 \mathrm{~h}$ after perigee: see second panel in Fig. 2b). The $B_{\mathrm{Z}}$ components all show negative values, but are much more complicated between about -3 and $+3 \mathrm{~h}$ relative to perigee. $\Delta B_{\text {model }}$ shows a "V" shape for this interval with minimum magnitude between -10 and $-30 \mathrm{nT}$ (for T01, $\sim-20 \mathrm{nT}$; for T96, $-30 \mathrm{nT}$; for T89, $\sim-10 \mathrm{nT}$ ) (see fourth panel in Fig. 2b). These effects arise because the magnetic field due to the ring current is oppositely directed to the main field in the equatorial inner magnetosphere (Sugiura et al., 1971). It is worth noting the bulges on the green lines (T01) and red lines (T96) at the boundary of ring current, which may be related to low-altitude cusp FACs and show clear signatures which are of the order of the observed external currents. It is apparent from these day and night-side passes that the models can predict either the smooth ring current or the short period FACs accurately, but do not always do so, and generally do not show the smaller-scale structure (transients) in the FACs.

Figure $2 \mathrm{c}$ and $\mathrm{d}$ shows a duskside and dawnside pass on 5 May 2004 and 4 December 2003, respectively. The conditions during the duskside pass are relatively active (IMF 
$B_{\mathrm{Z}}$ varies between about -5 and $5 \mathrm{nT}$, and SYM-H varies between about -10 and $-35 \mathrm{nT})$. The dawnside pass occurs under quiet conditions (IMF $B_{Z}$ varies between about -5 and $5 \mathrm{nT}$, and SYM-H varies between about -10 and $5 \mathrm{nT}$ ). The $B_{\mathrm{X}}, B_{\mathrm{Z}}$ and $\Delta B_{\text {model }}$ panels in Fig. $2 \mathrm{c}$ and $\mathrm{d}$ show similar features to those for the nightside pass. The $B_{Y}$ components, however, show a positive to negative polarity for the duskside pass and negative to positive polarity for the dawnside pass between about $-4 \mathrm{~h}$ and $+2 \mathrm{~h}$ relative to perigee; T96 estimated the biggest magnitude at the center of ring current crossing. It is also worth noting that there are some bulges on green lines (T01) and red lines (T96) at the boundary of ring current, which are related to region 2 FACs. Comparing the different phases of the Cluster crossings, we find all the components are smallest during the dayside pass and largest during the nightside pass. There is a clear asymmetry at both boundaries of the ring current during the dayside pass, which leads to a single bipolar signature at one boundary of the ring current associated with the low altitude FACs in the cusp region.

In a similar manner to the test performed by Woodfield et al. (2007) we compare the measured external field with the modelled ones. If one of the Tsyganenko models gave a perfect representation of the external magnetic field, the predicted traces (black, red, or green line) would be identical to the magenta lines. In Fig. 2 we find the magenta lines generally lie close to the three model lines for the whole interval but they show clear short bipolar signatures at the boundaries of the ring current crossing and similar monopolar signatures during the middle altitude cusp crossing (see Fig. 1). It is worth noting that the magenta lines are closest to the green line (T01) for the whole interval in each phase, which suggests that T01 achieves the best fit to the data.

Figure 3 shows an overview of the external magnetic field predicted by T01 at the tracks of Cluster S/C 1 for the whole eight years data (from orbit 93 on 1 February 2001 to orbit 1244 on 31 August 2008), together with the measured external field relative to the IGRF $\left(\boldsymbol{B}_{\mathrm{EO}}=\boldsymbol{B}_{\text {Observed }}-\boldsymbol{B}_{\mathrm{IGRF}}\right.$, as shown in Fig. 2). In this plot format each vertical strip is a section of an orbit: the $\mathrm{X}$-axis gives the orbit number, the $y$-axis is time relative to perigee and the colour scale is the value of $B_{\mathrm{XGSM}}, B_{\mathrm{YGSM}}, B_{\mathrm{ZGSM}}, \Delta B_{\text {model }}$ or $\Delta B_{\text {observed }}$ $\left(\Delta B_{\text {observed }}=\left|B_{\text {observed }}\right|-\left|B_{\text {IGRF }}\right|\right)$. The left column shows the output from T01 and the right column shows the measured external field $\left(\boldsymbol{B}_{\mathrm{EO}}\right)$. Note that in this figure (and also in Figs. 5 and 6) there are some regular and irregular data gaps in the measured field (white areas on the plots). The regular gaps, such as the ellipse-shaped gaps near perigee from the middle of February to the middle of March in each year, and the short white bars near +4 from the end of October to the beginning of July in 2002-2007, arise for various operational reasons. There are also missing data between orbit 719 (2 March 2005) and 745 (2 May 2005), which can be seen as a discontinuity in the colours shown in the plots.
Comparing the data and model panels of Fig. 3, we find that the observed external field is broadly similar to the T01 modeled external field (the predictions from T89 and T96 (not shown) show similar but weaker features to those from T01). For the whole eight year period, the $B_{\mathrm{X}}$ components are negative during pre-perigee crossing and positive during post-perigee, with "step-like" colour signatures near the perigee crossing at around $-1 \mathrm{~h}$ to perigee starting from each June to December. The $B_{Y}$ components are much weaker during the midnight sector perigee crossing between the middle of February and March and during the noon sector perigee crossing between the middle of August and October. Relative to perigee, they are negative (in blue) around $-2 \mathrm{~h}$, positive (in red) around $+1 \mathrm{~h}$, and negative around $+5 \mathrm{~h}$ between the middle of October and next February, and are positive (in red) around $-2 \mathrm{~h}$, negative (in blue) around $+1 \mathrm{~h}$, and positive around $+5 \mathrm{~h}$ relative to perigee from the middle of March to the middle of August. It is worth noting that the weaker values around $+3 \mathrm{~h}$ to perigee tend to move from about $+3 \mathrm{~h}$ to about $+2 \mathrm{~h}$ during the later years, which arises from the southward drop of the Cluster orbit as shown in Fig. 1.

From Fig. 3 we find that the T01 model predicts the ring current, which appears as the seasonally-dependent trough in the $B_{\mathrm{Z}}$ component and in $\Delta B_{\text {model }}$ around perigee: strongly negative in winter and spring (when perigee is on the nightside and passing close and through the ring current), and weakly positive or negative in summer and autumn (when perigee is on the dayside). The ring current crossing (between the boundaries of the trough represented by deep blue colour around the perigee) occurs between $\sim-0.5$ to $0.5 \mathrm{~h}$ around perigee at the beginning and end of the dataset and extends to $\sim-1.0$ to $1.0 \mathrm{~h}$ in the middle. In the orbits around 420, 600 and 780, dating from spring and summer in 2003, 2004 and 2005 respectively, the time taken to pass through the ring current is particularly long. The extension of the ring current does not change smoothly from orbit to orbit, but has a sawtooth shape at both boundaries at about +1 and $-1 \mathrm{~h}$ relative to perigee. Near both boundaries of the ring current, we find that there are small signatures (positive to negative or negative to positive in pre-perigee crossing and/or in post-perigee crossing) in the $B_{\mathrm{Y}}$ component, especially for the T01 prediction (see the example in the second panels in Fig. 2), which are associated with the region 2 FAC or cusp FAC. As an example, we show a region, highlighted by the black ellipse in each column, when the Cluster spacecraft were crossing the region 2 FAC. We find the FAC signature is increasingly prominent from T89, through T96 to T01. This is because the T89 model (not shown) does not include region 2 FAC, the T96 model (not shown) underestimates it and the T01 model (the left column in Fig. 3) performs better and closest to the measured FAC (the right column in Fig. 3).

Comparing the modelled, external magnetic field from the different models, we find that the $B_{X}$ component was stronger, and the $B_{\mathrm{Y}}$ and $B_{\mathrm{Z}}$ components and $\Delta B_{\text {model }}$ were 
T01
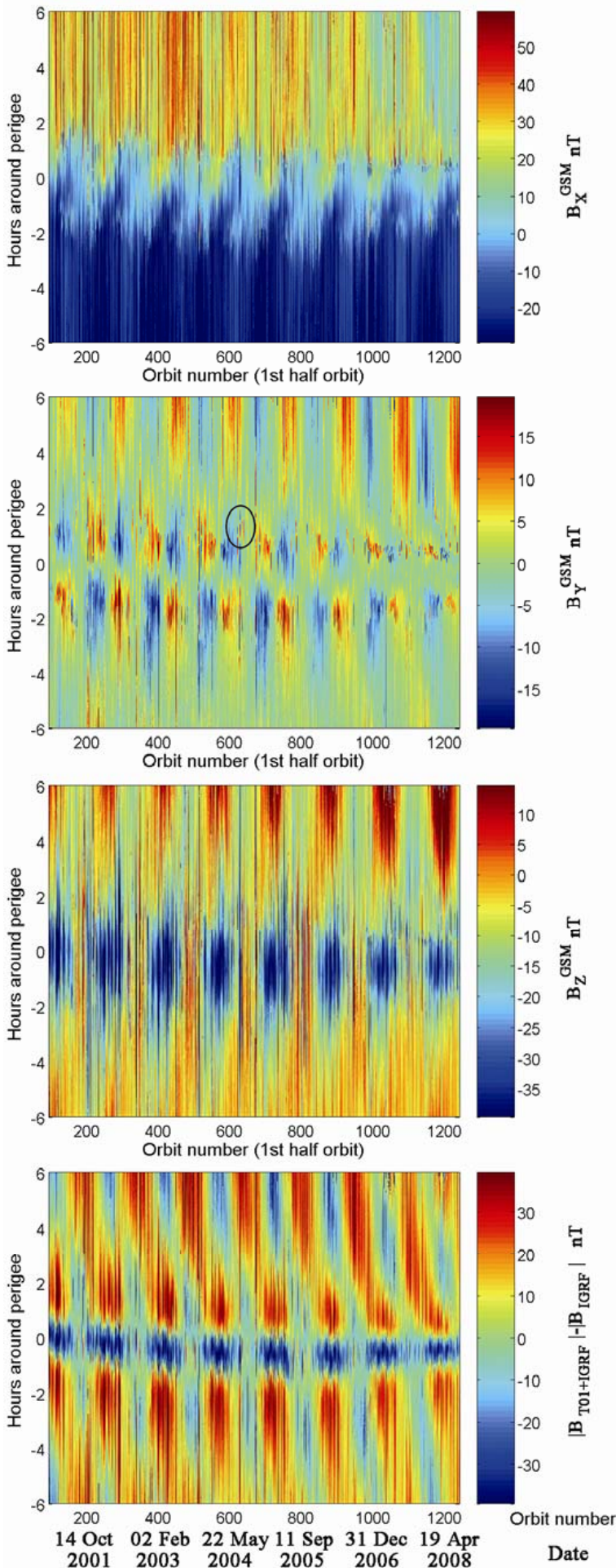

Cluster 1 - IGRF
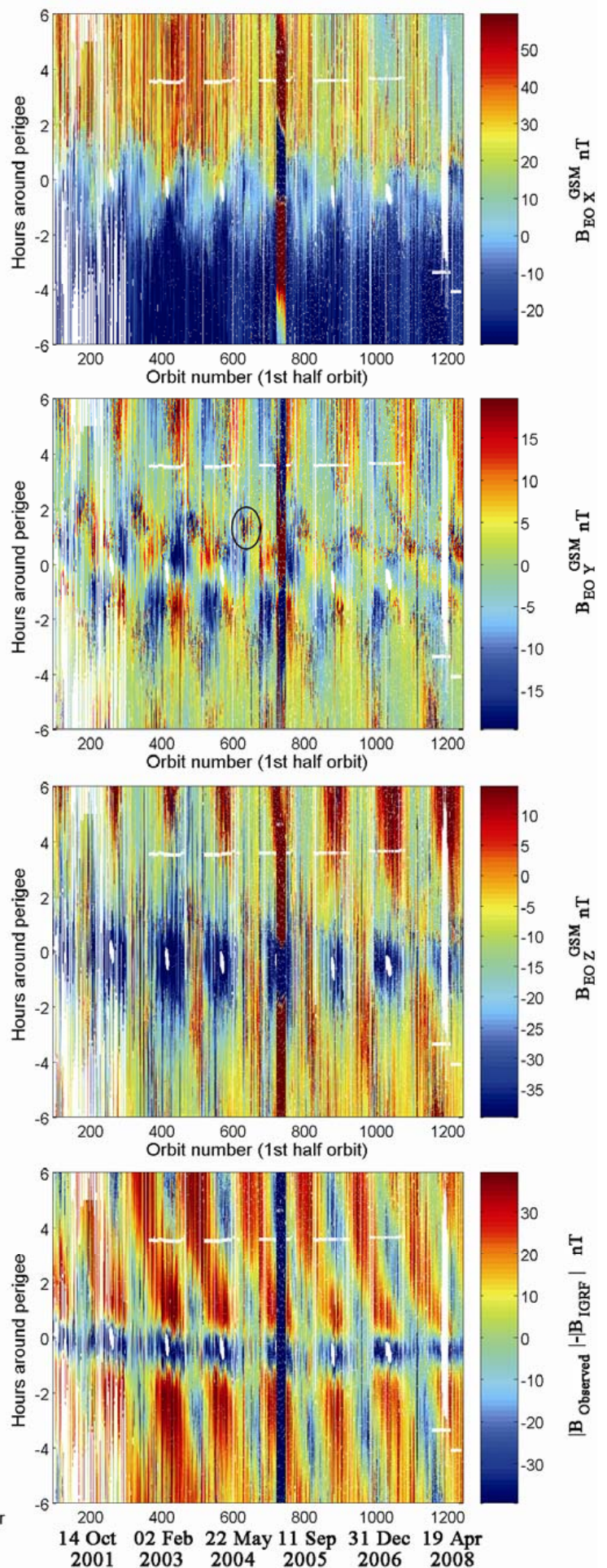

Fig. 3. Plots of the three components and magnitude of external magnetic field predicted by T01 and measured by Cluster S/C 1 (minus IGRF) at the tracks of Cluster S/C 1 for the whole eight years data. Each vertical strip is a section of an orbit - the $\mathrm{x}$-axis is the orbit number, $\mathrm{y}$-axis is time relative to perigee and the colour scale is the value of $B_{\mathrm{XGSM}}, B_{\mathrm{YGSM}}, B_{\mathrm{ZGSM}}$, and $\Delta B_{\text {model }}$ or $\Delta B_{\mathrm{observed}}$ (each row), for the panels of the left column from T01 and the measured (minus IGRF) for the right column. 
weaker for the T01 model compared with the others. Note that the time variation in input conditions causes significant deviations in $B$ (see detailed discussion in Sect. 4.1). The residuals of the Cluster S/C 1 observations with the IGRF model (the observed external field, $\boldsymbol{B}_{\mathrm{EO}}=\boldsymbol{B}_{\text {Observed }}-$

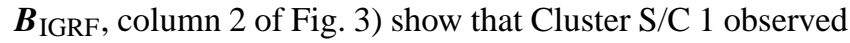
the ring current magnetic field around perigee and observed prominent sharp, bipolar signatures in all three components before perigee $(\sim-1.5 \mathrm{~h})$ and after perigee $(\sim+1 \mathrm{~h})$ (also seen in Fig. 2a, b, c and d). The location of these bipolar features is closely aligned with the boundary of the ring current during the perigee pass. There are some sharp signatures around 5 and $6 \mathrm{~h}$ after perigee (see Fig. 2a). These are suggestive of crossings through regions of enhanced plasma pressure and might be contributed to by the current in the cusp region. Support for this hypothesis comes from the sharp increase in the electron and ion flux observed by RAPID (not shown here). These sharp changes in the residual component fields are mainly monopolar in shape and are much more complicated at the cusp region (about 5 to $6 \mathrm{~h}$ away from perigee).

Woodfield et al. (2007) suggested the bipolar signatures that Cluster sees close to the ring current are the result of passing through, or close to, a region $2 \mathrm{FAC}$. The components and magnitude of the observed external fields are indeed consistent with the spacecraft passing a tube of current, albeit including additional filamentary structure. During the spacecraft cusp crossing, the residuals show monopolar structures with a decrease in magnetic field magnitude, which might indicate that the spacecraft passes through or close to a current system not parallel to the main magnetic field, poorly estimated by the Tsyganenko models (magnetic field depressions associated with the cusps were extensively discussed by Tsyganenko and Russell, 1999, and Tsyganenko, 2009).

\section{Results for the measured residuals}

\subsection{Comparison between Cluster data and the different Tsyganenko models}

Figure 4 shows the data residuals after differencing the Cluster S/C 1 observations with the predictions from the T89 (black), T96 (red) and T01 (green) models $(d \boldsymbol{B}=$ $\boldsymbol{B}_{\text {Observed }}-\boldsymbol{B}_{-\mathrm{T}}-\boldsymbol{B}_{\text {IGRF}}$ ) respectively for: (a) the nightside pass on 13 February 2004, (b) the dayside pass on $2 \mathrm{Au}-$ gust 2004, (c) the duskside pass on 8 May 2004 and (d) the dawnside pass on 4 December 2003. From top to bottom the panels in each of Fig. $4 \mathrm{a}-\mathrm{d}$ are for the $d B_{\mathrm{XGSM}}, d B_{\mathrm{YGSM}}$, $d B_{\mathrm{ZGSM}}$ and the residuals in the magnitude of the field. As in Fig. 2, the grey shaded region shows the ring current extent as defined by the energetic particle flux. The residuals are almost zero except during the crossings of the ring current, region 2 FAC and cusp current regions. These residuals measure the degree to which the model currents match the measured behaviour on these days. The residuals show that the models overestimate or underestimate the contributions of the ring current, underestimate the contributions of the FAC, especially the region $2 \mathrm{FAC}$, and do not identify the contributions from the cusp current (about $5 \mathrm{~h}$ after perigee in Fig. 4a). The offset between the data and the predicted value of the magnetic field within the region 2 FAC reaches a maximum of approximately $50 \mathrm{nT}$ (e.g. Fig. 4a), and a maximum of about $20 \mathrm{nT}$ within the ring current. These differences also depend on the global orbital orientation, represented by each pass in Fig. 4a-d (dayside, nightside, and both flanks). The sharp signatures, associated with the region 2 FAC or the cusp FAC, are shown in all three components for the nightside pass, mainly in $B_{\mathrm{Y}}$ with weaker signatures in $B_{\mathrm{X}}$ for the dayside pass, mainly in $B_{\mathrm{X}}$ with weaker signatures in $B_{\mathrm{Y}}$ for the duskside pass and mainly shown in $B_{X}$ with weaker signatures in $B_{\mathrm{Y}}$ and $B_{\mathrm{Z}}$ for the dawnside pass. This might reflect the distribution of FACs at different local times. Comparing the residuals from the different models, we find that they all show similar features during the whole interval, particularly in the $B_{Y}$ component. From the residual of the magnitude, we find that for these cases, T96 overestimated the ring current (positive residuals) and the other models underestimated it (negative residuals). None of the models identify the cusp current.

Comparing the residuals relative to the Tsyganenko models with those relative to IGRF only (in Fig. 2), we find the Tsyganenko models estimate the ring current well, but give a poor estimate of the field strength arising from region 2 FAC. The cusp currents remain largely un-modeled. Thus, we find that, while clear signatures of FACs and cusp persist in the traces in Fig. 4, the deviations in the ring current are different for each model. It is interesting to note that in most of the regions sampled, the $B_{\mathrm{Y}}$ component shows very similar residuals from the 3 models. Additionally, in the dawnside and dayside regions (Fig. $4 \mathrm{~b}, \mathrm{~d}$ ), $B_{\mathrm{X}}$ shows similar residuals for all models. These effects are reflected in the trends seen over the whole mission as discussed below (in Fig. 5).

Figure 5 shows the residuals from the comparisons of Cluster S/C 1 observations with T89, T96 and T01 models $\left(d \boldsymbol{B}=\boldsymbol{B}_{\text {Observed }}-\boldsymbol{B}_{-\mathrm{T}}-\boldsymbol{B}_{\mathrm{IGRF}}\right)$ for the whole eight years of data in a similar format to Fig. 3. Residuals are shown from T89, T96 and T01 respectively from left to right columns. The T89 and T01 models generally underestimated the ring current in the earlier 4 or 5 years, while the T96 overestimated it almost for the whole 8 years. For T89, the $d B_{\mathrm{X}}$ component in GSM coordinates deviates much more than the others, so that the pre-perigee crossing (in the Southern Hemisphere) is underestimated and the post-perigee crossing (in the Northern Hemisphere) is overestimated. The $d B_{Y}$ component is similar for all the models, and the behaviour is governed more by the external conditions, but is progressively underestimated during the later years. For T96, $d B_{X}$ and $d B_{\mathrm{Y}}$ vary between the results for T89 and T01. $d B_{\mathrm{Z}}$ shows similar, but more complicated, behaviour. For T01, all of the 

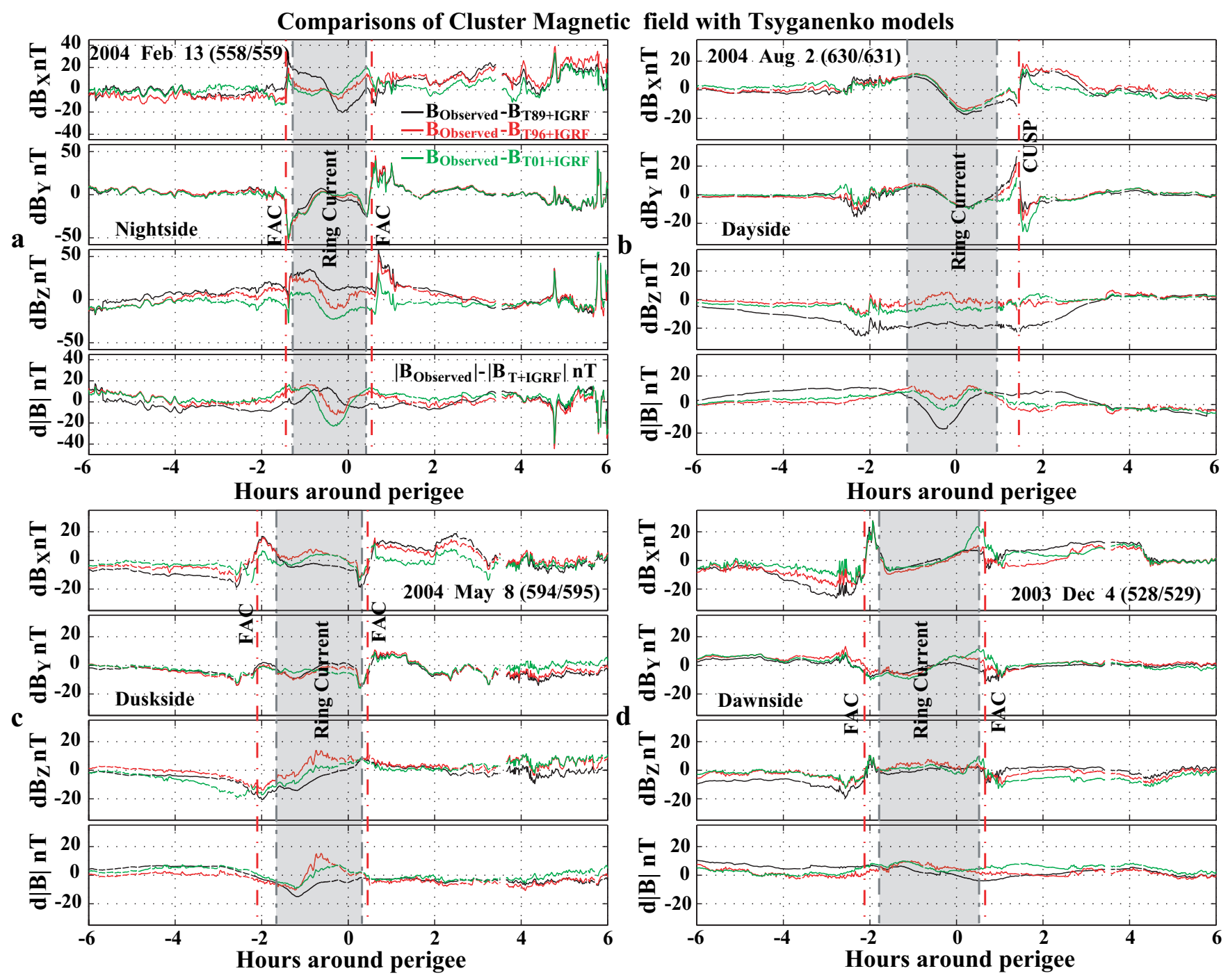

Fig. 4. Residuals from the comparisons of Cluster S/C 1 observations with T89 (black), T96 (red) and T01 (green) and IGRF (magenta) models for the nightside pass on 13 February 2004 (a), dayside pass on 2 August 2004 (b), duskside pass on 8 May 2004 (c) and dawnside pass on 4 December 2003 (d). From top to bottom panels of Fig. 5a-d are for the $d B_{\mathrm{XGSM}}, d B_{\mathrm{YGSM}}, d B_{\mathrm{ZGSM}}$ and the magnitude of the field. The black vertical lines show the ring current region crossing.

components of the deviations are much smaller than from the other models, indicating that this model best fits the data. The time-varying input parameters return a time-varying model field since the magnetospheric response is partially contained in T01. Although this time varying input provides a better fit to the data the residuals have less meaning. Nevertheless, it is clear that the features indicated by the line plots (Fig. 4), for FAC and cusp, persist throughout. The T01 deviations revealed are weaker during the later years, which might be because of the approach to solar minimum with reducing geomagnetic activity. (The IMF conditions and SYM-H index (not shown) confirm that conditions are broadly active during the first four and a half years (from orbit 93 to $\sim 800$ ) and quiet in the later years (from orbit $\sim 800$ to 1244 )).
As mentioned above, the lines of apsides of the Cluster spacecraft drop southward in the later years,. In order to investigate the regions encountered at different times due to this dropping, we compare in detail three months (January to March) of results from 2004 and 2007. Figure 6a and $b$ shows the residuals from the comparisons of Cluster S/C 1 observations with the T89, T96 and T01 models for the orbits 540-578 and 1001-1038, which are from 1 January to 31 March in 2004 and 2007, respectively. Figure 6a and $\mathrm{b}$ shows clear bipolar signatures (sharp blue/red trends or red/blue trends) corresponding to the contributions of region $2 \mathrm{FAC}$ at $\sim 1.0 \mathrm{~h}$ either side of perigee for the whole three months of 2004 , and $\sim-2.0 \mathrm{~h}$ and $0.5 \mathrm{~h}$ relative to the perigee for the whole three months of 2007 (most clear in the 

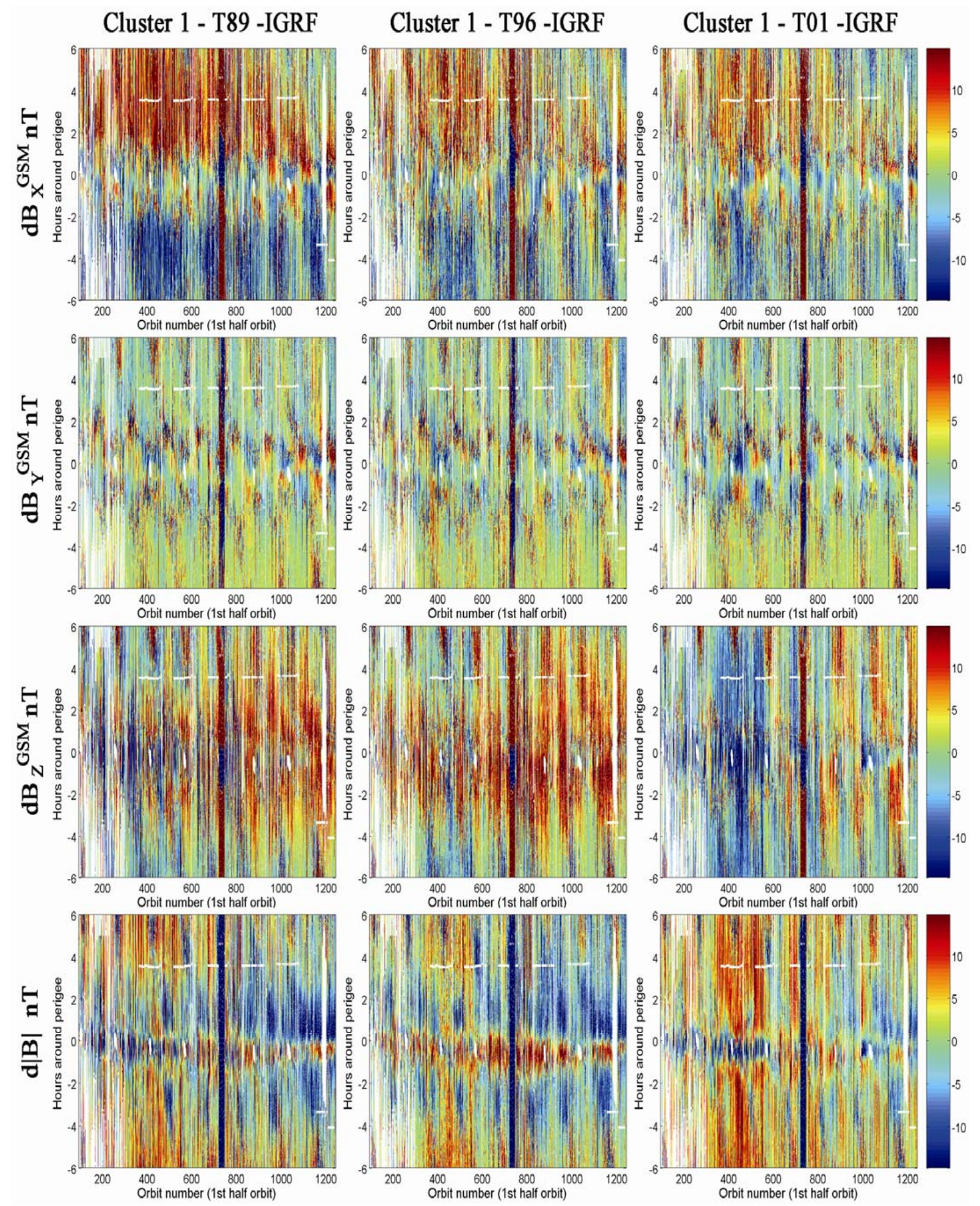

Fig. 5. Plots of the residuals from the comparisons of Cluster S/C 1 observations with T89, T96 and T01 models for the whole eight years data. Each vertical strip is a section of an orbit - the $\mathrm{x}$-axis is the orbit number, $\mathrm{y}$-axis is time relative to perigee and the colour scale is the value of $d B_{\mathrm{XGSM}}, d B_{\mathrm{YGSM}}, d B_{\mathrm{ZGSM}}$ (each row), the panels from the left to right columns are the residuals from T89, T96 and T01, respectively. 

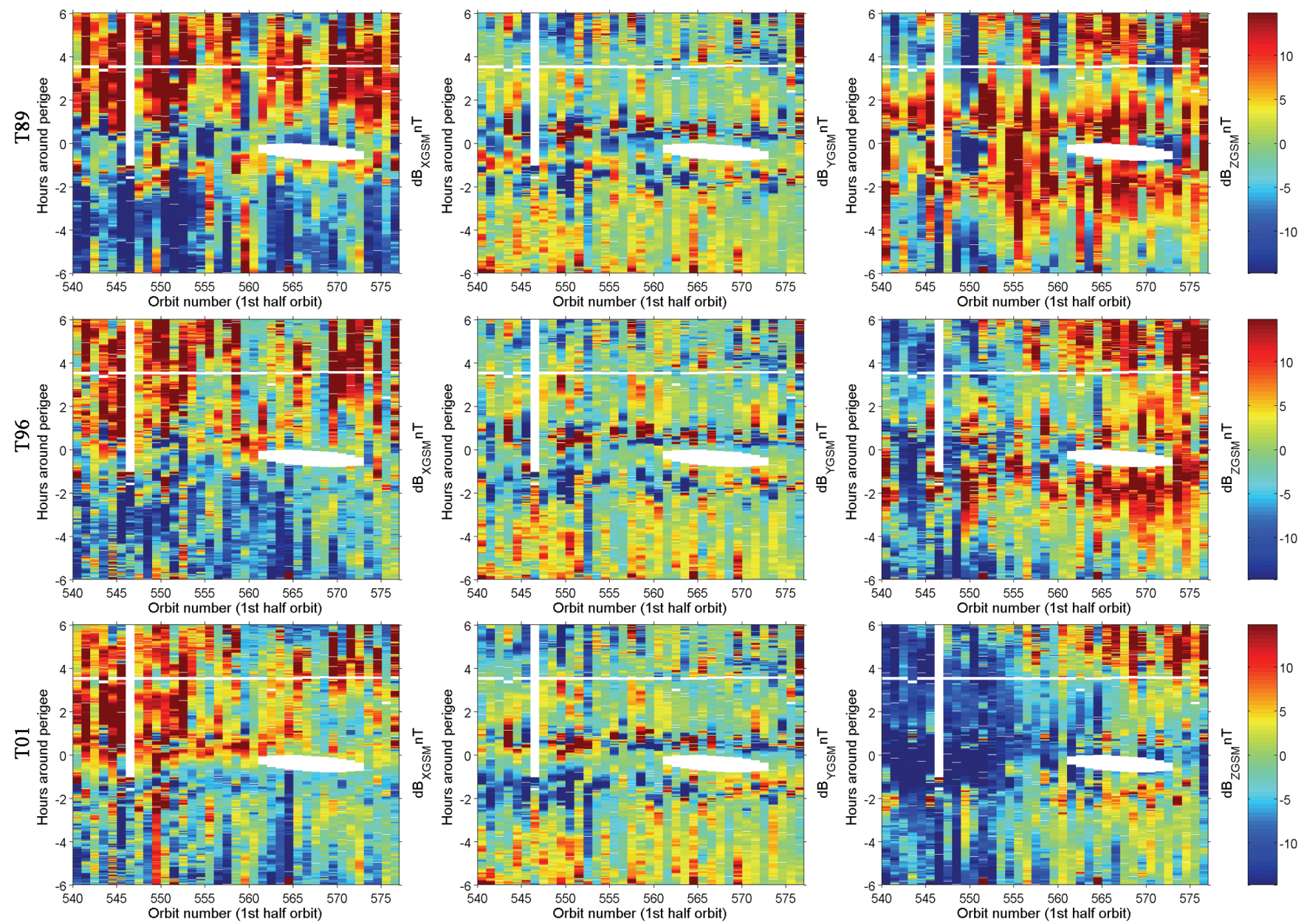

Fig. 6. Plots of the residuals from the comparisons of Cluster S/C 1 observations with T89, T96 and T01 models for the orbits 553-566 and 1014-1026, which are from 1st to 29 (28) February in 2004 and 2007, respectively.

$B_{Y}$ component). These tend to show underestimated signatures around $\pm 1.0 \mathrm{~h}$ of perigee in $d B_{\mathrm{Z}}$. The T89 model, however, does not include (and the T96 model does not well reproduce) the region $2 \mathrm{FACs}$, resulting in larger residuals. The deviations also show that there are underestimated signatures (redder colour, especially in $d B_{\mathrm{Z}}$ ), reflecting the contributions of the cusp current system, between $\sim+3.0$ and $+6.0 \mathrm{~h}$ for the whole three months of 2004, and between $\sim+1.0$ and $+3.0 \mathrm{~h}$ for the whole three months of 2007, again a result of the progressive dropping southward of the Cluster orbit as shown in Fig. 1.

\subsection{Comparisons between the 4 Cluster spacecraft}

In order to further investigate the cause of the bipolar signatures we have attributed to FACs in the previous section we make use of the multi-spacecraft nature of the Cluster mission. By studying the individual spacecraft residuals from T01 for the whole eight years of data (not shown), we find that the residuals are almost the same at all 4 spacecraft. There are some clear differences in the $d B_{\mathrm{X}}$ and $d B_{\mathrm{Y}}$ components in later years, as a consequence of the larger spacecraft separations. At the boundary of the ring current in the later years, the Cluster S/C 2 observed the strongest signatures, followed by S/C 1; S/C 3 and 4 measured almost the same features. This might be because Cluster S/C 2 is always located outermost, S/C 1 follows and S/C 3 and 4 are located innermost (and move almost together: see, for example, the configurations in Fig. 1), resulting in each spacecraft crossing or passing close to different parts of the FAC.

Figure 7 demonstrates the effects of the different spacecraft separations and orientations on the magnetic field residuals. Figure $7 \mathrm{a}$ shows the nightside passes on 13 February 2004 (small average separation, $\sim 160 \mathrm{~km}$ ) in the left-hand panels and on 12 February 2007 (large average separation, $\sim 1600 \mathrm{~km}$ ) in the right-hand panels. The left and right panels in Fig. 7b similarly show the dayside passes on $2 \mathrm{Au}$ gust 2004 (middle average separation, $\sim 720 \mathrm{~km}$ ) and on 16 

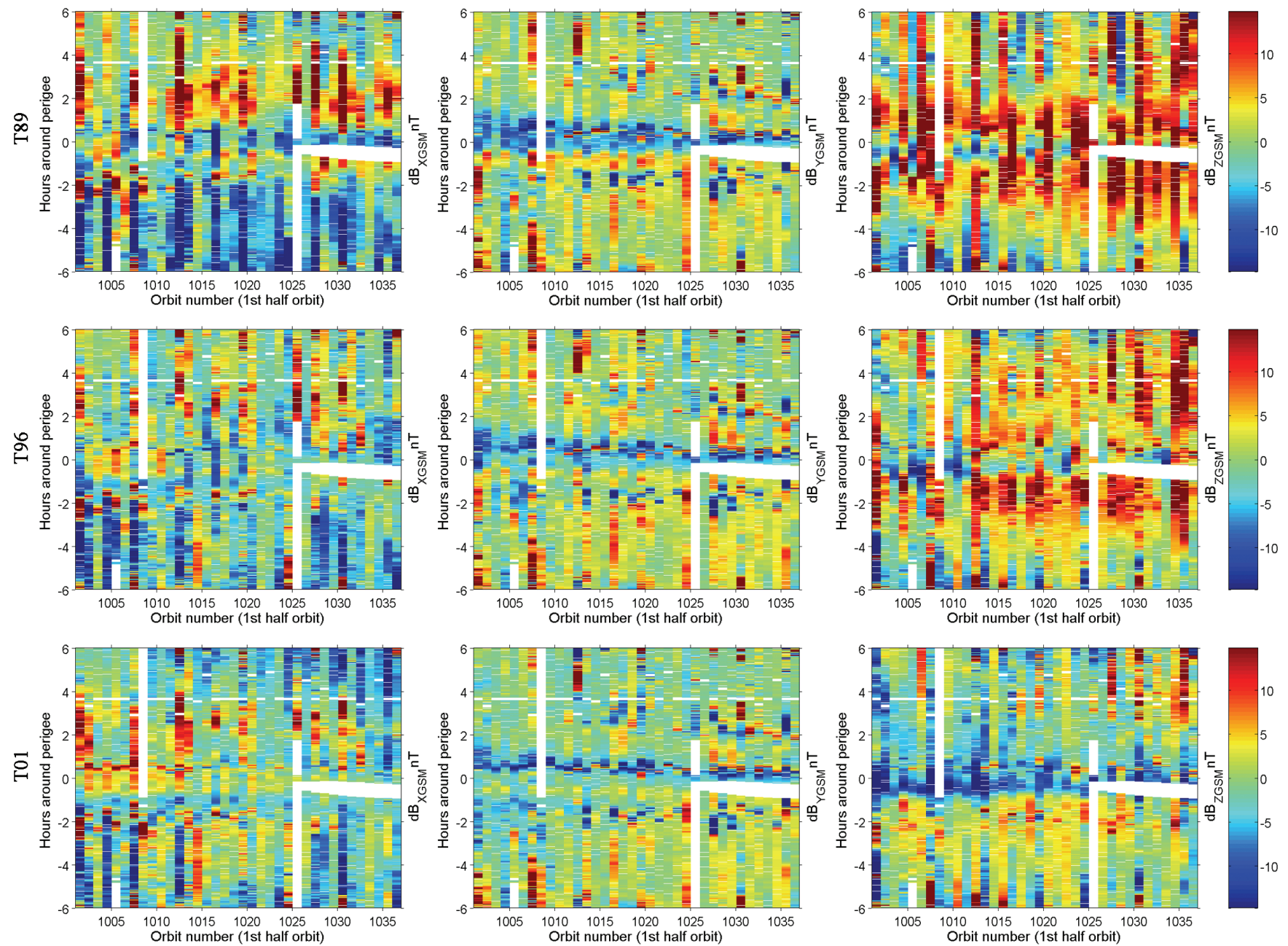

Fig. 6. Continued.

August 2007 (large average separation, $\sim 2058 \mathrm{~km}$ ), respectively. In the same format, Fig. 7c shows the pair of flank passes on 8 May 2004 and 4 December 2003. In each of the plots in Fig. 7, the top four panels are residuals (datamodel) of magnetic field in GSM coordinates and the fifth panel shows the magnetic field magnitude from the 4 Cluster spacecraft for the two orbits. In addition, at the bottom of each plot, the normal components of the residuals averaged over the 4 Cluster spacecraft are shown. These are the projections of $d \boldsymbol{B}_{\text {avg }}$ and $\boldsymbol{B}_{\mathrm{EO}-\text { avg }}$ onto the normal direction of the current tubes for each FAC: $d \boldsymbol{B}_{\mathrm{Navg}}=d \boldsymbol{B}_{\mathrm{avg}} \bullet \hat{n}_{v}$ and $\boldsymbol{B}_{\mathrm{EO}-\mathrm{Navg}}=\boldsymbol{B}_{\mathrm{EO}-\mathrm{avg}} \bullet \hat{n}_{v}$. In $\boldsymbol{B}_{\mathrm{EO}-\mathrm{Navg}}$, the bipolar signature is attributed to a current tube crossing by the spacecraft, while in $d B_{\mathrm{Navg}}$, it is due to the contributions of the underestimated part of the observed current tube. This means the model performs better when the offset is larger between $d \boldsymbol{B}_{\mathrm{Navg}}$ and $\boldsymbol{B}_{\mathrm{EO}-\mathrm{Navg}}$. The $\mathrm{x}$-axis in these lower panels is time relative to the perigee for each orbit.
From Fig. 7, we find all four spacecraft observed the same signatures when they were at small separation and observed similar signatures, successively with small differences, when they were at large separation. The bipolar signatures, which are thought to be the contributions from region 2 FACs, are clearly shown in the residuals from all the four spacecraft, the differences between the spacecraft to spacecraft give an indication of FAC spatial extent. In Fig. 7a there are also some complicated structures between +1.8 and $+2.8 \mathrm{~h}$ of perigee in the February 2007 pass (about three hours earlier than the one in February 2004). This may suggest the Cluster spacecraft are crossing the cusp current system at mid to high altitudes.

Since all four spacecraft typically sample the region 2 FACs, we may apply four-spacecraft timing analysis (Russell et al., 1983; Dunlop and Woodward, 1998) to calculate the motion and scale of the FACs observed by Cluster, using the known tetrahedral spacecraft configuration. The results are given in Table 2, which are similar in each case and 
Comparisons of Cluster Magnetic field with T01 model
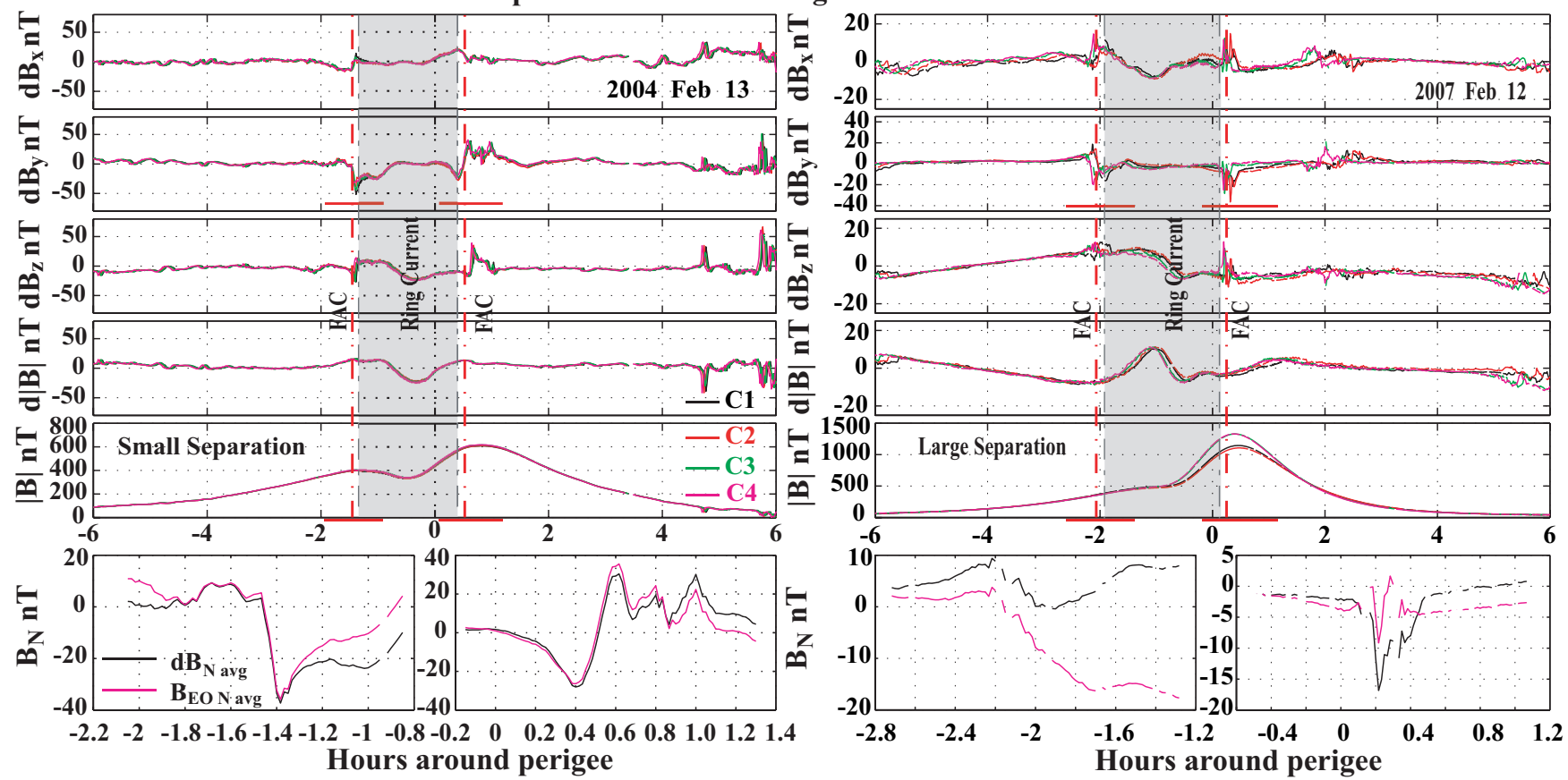

Hours around perigee

Comparisons of Cluster Magnetic field with T01 model

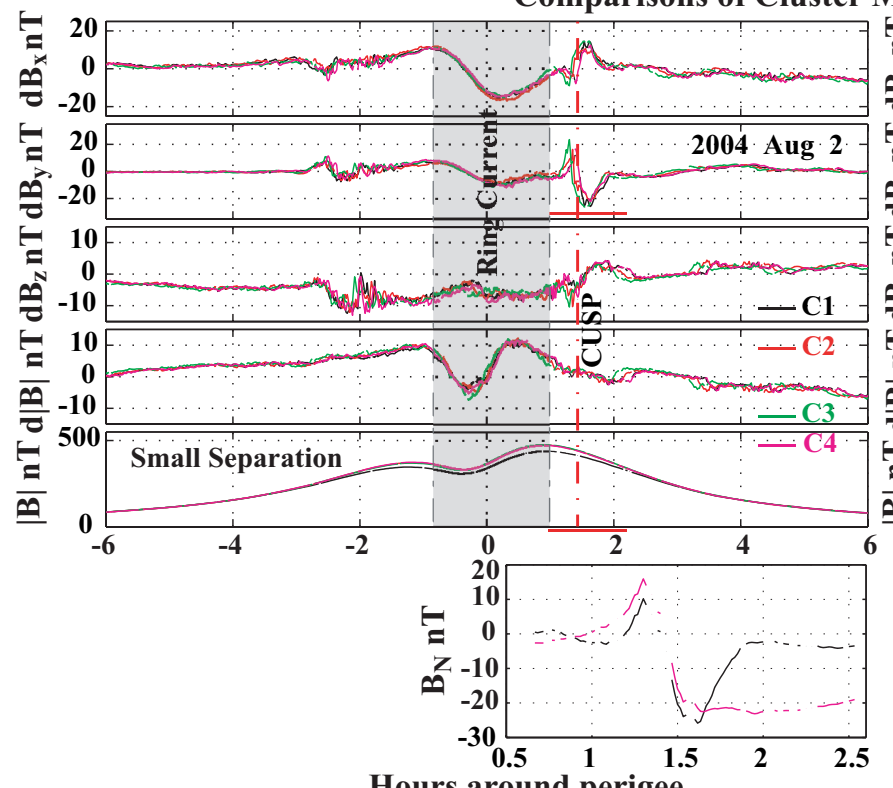

Hours around perigee

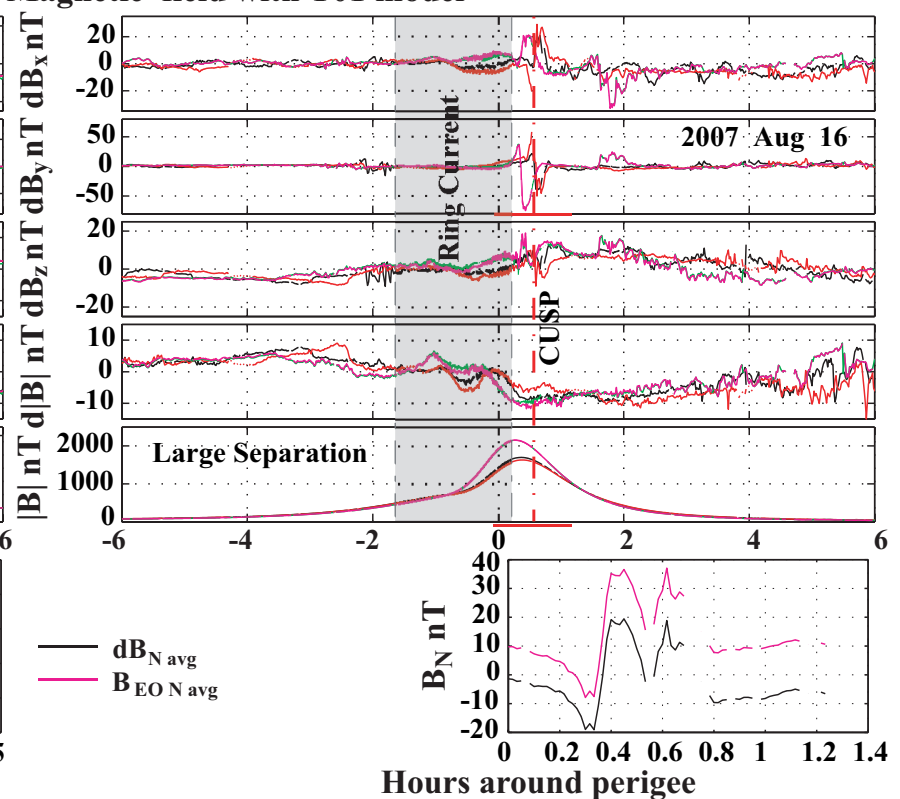

Fig. 7. Results from six orbits with different separations and different orientations of Cluster 4-spacecraft on 13 February 2004 and 12 February 2007 (a, in small and large separation, nightside pass), on 2 August 2004 and 16 August 2007 (b, in middle and large separation, dayside pass), and on 8 May 2004 and 4 December 2003 (c, in small separation, both flanks pass), together with the average residual normal

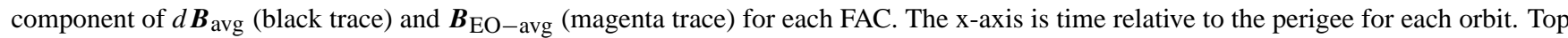
four panels are residuals (data-model) of magnetic field in GSM coordinates. Fifth panel shows the magnetic field magnitude from the 4 Cluster spacecraft for the two orbits.

give the average direction of the magnetic field from the 4 Cluster spacecraft, $\hat{b}_{\text {avg }}$ (the third column of Table 2) and the normal of the current tubes, $\hat{n}_{v}$ (the fourth row of Table 2).
Angles between $\hat{b}_{\text {avg }}$ and $\hat{n}_{v}$ range from 86.8 to $111.5^{\circ}$ (the sixth column of Table 2), suggesting that these structures are likely to be FACs, and are crossed perpendicularly. From 


\section{Comparisons of Cluster Magnetic field with T01 model}
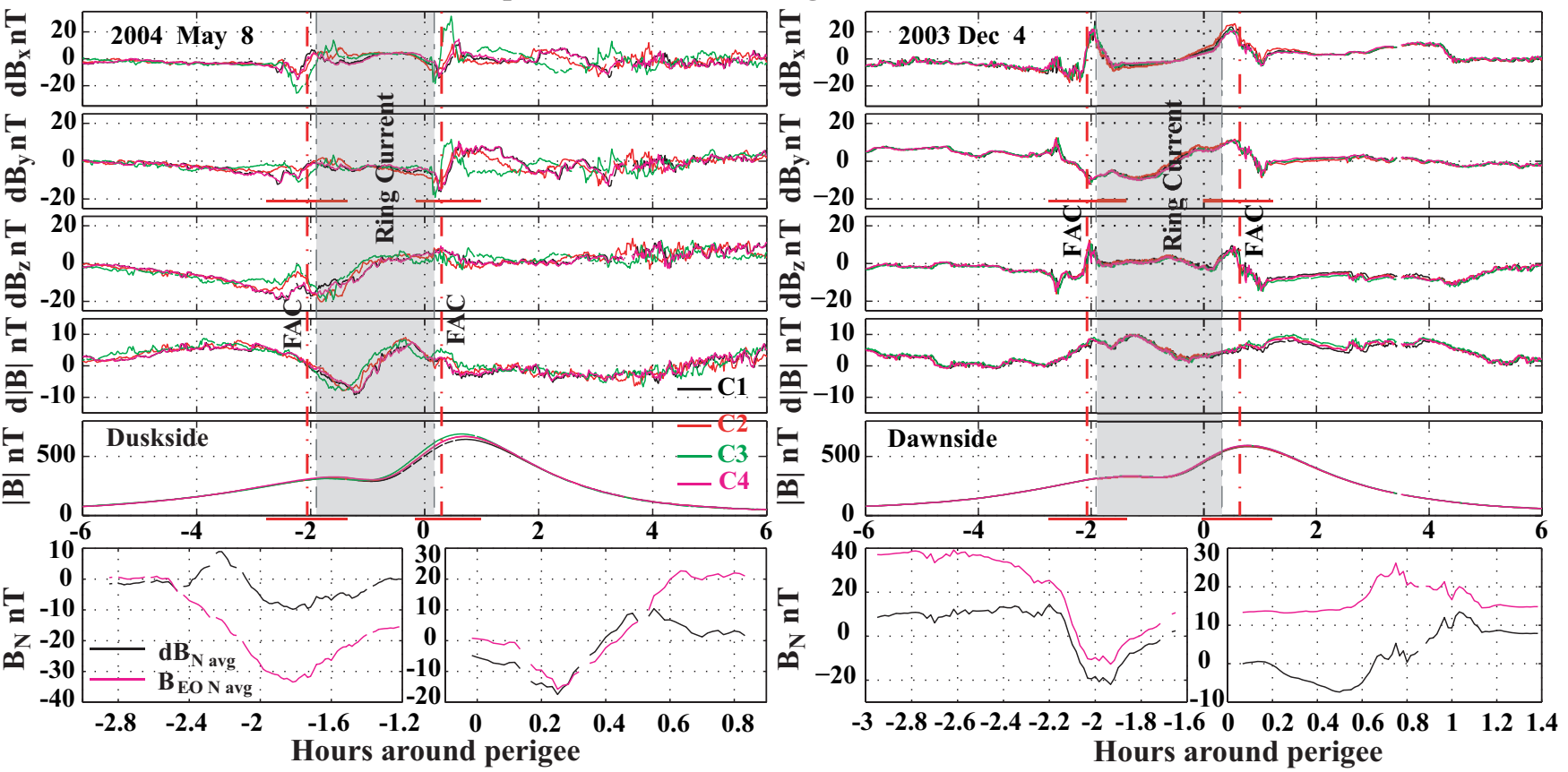

Fig. 7. Continued.

Table 2. Catalogue of the average magnetic field and FACs motion for the FACs observed by Cluster spacecraft in the orbit shown in Fig. 1, together with the angle between the average magnetic field and the FACs motion. The directions $\left(\boldsymbol{n}_{v}\right)$ and the speeds $(|\boldsymbol{V}|)$ of the motion are obtained from four-spacecraft techniques, and the size $\left(D_{\mathrm{FAC}}\right)$ of each FAC observed by Cluster was estimated by using the velocity and the duration of the whole bipolar signature of each FAC.

\begin{tabular}{ccccccc}
\hline Date & Hours to perigee & $\begin{array}{c}\boldsymbol{B}_{\text {avg GSM }} \\
\text { X, Y, Z }\end{array}$ & $\begin{array}{c}\boldsymbol{n}_{\mathrm{V}} \text { GSM } \\
\mathrm{X}, \mathrm{Y}, \mathrm{Z}\end{array}$ & $\begin{array}{c}|\mathbf{V}| \\
(\mathrm{km} / \mathrm{s})\end{array}$ & $\begin{array}{c}\text { Angle } \\
\left({ }^{\circ}\right)\end{array}$ & $\begin{array}{c}D_{\mathrm{FAC}} \\
\left(R_{\mathrm{E}}\right)\end{array}$ \\
\hline \multirow{2}{*}{ 13 Feb 2004 } & $\sim-1.4$ & $-0.87,-0.48,-0.09$ & $-0.24,0.92,-0.31$ & 0.73 & 101.7 & 0.21 \\
2 Aug 2004 & $\sim+0.6$ & $0.95,0.29,-0.12$ & $-0.39,0.92,-0.06$ & 0.77 & 96.8 & 0.54 \\
& $\sim+1.5$ & $-0.68,-0.45,-0.58$ & $-0.54,0.84,0.09$ & 0.13 & 93.5 & 0.06 \\
8 May 2004 & $\sim-2.2$ & $-0.24,0.84,0.50$ & $-0.41,-0.73,0.55$ & 0.21 & 104.0 & 0.15 \\
& $\sim+0.4$ & $0.29,-0.50,-0.82$ & $-0.94,-0.32,0.13$ & 0.17 & 102.5 & 0.09 \\
4 Dec 2003 & $\sim-2.5$ & $0.04,-0.92,-0.39$ & $-0.76,0.18,-0.63$ & 0.42 & 86.8 & 0.25 \\
& $\sim+0.6$ & $-0.00,0.80,-0.60$ & $0.20,-0.57,-0.80$ & 0.53 & 89.0 & 0.32 \\
12 Feb 2007 & $\sim-2.1$ & $-0.72,-0.36,-0.59$ & $0.88,0.01,-0.47$ & 1.42 & 111.5 & 0.43 \\
16 Aug 2007 & $\sim+0.2$ & $0.88,0.45,-0.14$ & $-0.24,0.97,-0.03$ & 0.35 & 102.8 & 0.17 \\
& $\sim+0.4$ & $-0.79,-0.18,-0.58$ & $-0.34,0.71,0.62$ & 0.22 & 102.5 & 0.12 \\
\hline
\end{tabular}

Table 2, we also find that for nightside passes (the FACs on 13 February 2004 and 12 February 2007) the directions of motion, along the components of $\hat{n}_{v}$, are southward and tailward and strongly duskward (eastward), except for the FAC at $\sim-2.1 \mathrm{~h}$ to perigee on 12 February 2007 which is southward and duskward, but dominated by a sunward motion. For the dayside passes (the FACs on 2 August 2004 and 16 August 2007), the implied directions of motion are all northward and tailward with very strong duskward (east- ward) components. For the duskside pass (the FACs on 8 May 2004), the implied directions of motion are all dawnward (westward), northward and tailward. For dawnside pass (the FACs on 4 December 2003), the implied direction of motion is duskward, dominated by southward and tailward components for the FAC at $\sim-2.5 \mathrm{~h}$ to perigee and dawnward and sunward, dominated by a southward component for the $\mathrm{FAC}$ at $\sim+0.6 \mathrm{~h}$ to perigee. 
The timing analysis is calculated relative to the spacecraft motion during the crossing of the structure. The FAC speeds, $|\mathrm{V}|$ in Table 2, range from about $0.22 \mathrm{~km} / \mathrm{s}$ to $1.42 \mathrm{~km} / \mathrm{s}$, suggesting that the structures are very stable and are almost stationary during the Cluster crossings. Assuming a cylindrical current tube we can estimate the maximum current tube size (transverse thickness), $D_{\mathrm{FAC}}$ using $D_{\mathrm{FAC}}=|V| \cdot \Delta t$ where $\Delta t$ is the duration of the whole bipolar signature surrounding each FAC. $D_{\text {FAC }}$ ranges (see Table 2) from about 0.17 to $0.54 R_{\mathrm{E}}$ for the region 2 FACs, and from about 0.06 to $0.12 R_{\mathrm{E}}$ for the cusp FACs. We project the average residuals of $d \boldsymbol{B}_{\text {avg }}$ and $\boldsymbol{B}_{\mathrm{EO}-\text { avg }}($ defined earlier) from the 4 spacecraft onto the relative normal directions $\hat{n}_{v}$ for these FACs. The results show clear bipolar structures (see the lower panels in Fig. $7 \mathrm{a}, \mathrm{b}$ and c) with a peak-to-peak magnitude of $\sim 50$ and $\sim 70 \mathrm{nT}$ for the FACs on 13 February 2004, and of $\sim 20$ and $\sim 25 \mathrm{nT}$ for the FACs on 12 February 2007. This also suggests the residuals are much weaker in the later years.

\section{Discussion}

The comparisons of eight years of magnetic field data from the 4 Cluster spacecraft with the predictions from Tsyganenko models confirm the frequent occurrence of sharp, bipolar features in the residual values (data minus model) in all three GSM magnetic field components, which were attributed to region 2 FAC by Woodfield et al. (2007). These bipolar signatures place the observed FACs on or near the boundary of the ring current. The morphology of the Birkeland current system suggested by Iijima and Potemra (1976) closes the region 2 system via the ring current; the ring current can extend from -65 to $65^{\circ}$ in latitude all over the evening and the post-midnight sector (Vallat et al., 2005). It is therefore possible that the FACs we have observed were indeed part of the region 2 current circuit (Woodfield et al., 2007), or the region 2 FACs located at the boundary of the ring current. This is in agreement with Vallat et al. (2005) where the authors used the four Cluster spacecraft to estimate the current using the curlometer technique (e.g. Dunlop et al., 2002). A curlometer analysis has not been included here since the quality is expected to be low in view of the spacecraft configuration in some of the relevant regions in these orbits. Nevertheless, such a study is being attempted through improvements to the curlometer technique.

The quasi-static model (T89) takes into account the tail current, ring current and Chapman-Ferraro currents, but does not include the larger scale region 1 and 2 FAC. The dynamic models (T96 and T01) take a detailed approach to modelling the region 1 and 2 Birkeland currents, including the change of ionospheric latitude with activity, dipole tilt-related deformation and the observed day-night asymmetry (Tsyganenko, 1996, 2002a). It is a difficult task to model these FAC systems realistically, however, and any attempt to include them in a global model is almost certain to be a simplification as a matter of necessity. In general, we find that T96 and T01 model the location of the observed FAC system signatures well but the magnitude and fine structure are less well reproduced, as there are additional filamentary structures embedded within each FAC. It is unrealistic to expect the model to be able to estimate accurately the detailed structure. It is of prime concern, however, to obtain a realistic prediction of the magnitude of the effect the FAC system has on the magnetic field. As the individual events showed, the intensity of the magnetic field perturbation due to the FAC system varies greatly. This increases the need for accurate and large data sets to be used in generating a model. The results presented here demonstrate the need for empirical models of the external field to better exploit Cluster magnetic field measurements, especially in the Birkeland current region.

The time that the FACs are encountered with respect to perigee alters over the course of a year as the plane of the Cluster orbit precesses through $24 \mathrm{~h}$ in local time (see Woodfield et al., 2007, Fig. 13). It would be expected, given the day-night asymmetry mentioned above, that orbits with a dayside perigee would observe any region 2 current signatures further towards the poles (see, for example, Tsyganenko (2002a), Fig. 4). This is in agreement with our results; the FAC signatures occur further away from perigee from about July to December.

The separations of the 4 Cluster spacecraft vary over the mission from about $100 \mathrm{~km}$ to $2-3 R_{\mathrm{E}}$, which allows us to make spatial comparisons during similar external conditions. At small separations, all the spacecraft observed the same features with clear bipolar signatures resulting from the region 2 FACs (see Fig. 7). The 4-spacecraft technique (timing analysis) works very well for calculating the normal direction and scale of these FACs and the results confirm the presence of FACs. The deviations in the normal directions show a positive to negative bipolar signature at the pre-perigee crossing and a negative to positive bipolar signature, with some turbulence at the end, during the post-perigee crossing (see the bottom panels in Fig. 7). This indicates that the Cluster spacecraft array is crossing one or two poleward FACs in the pre-midnight, post-noon and dusk sectors, and tailward FACs in the dawn sector, which confirms the statistical distribution of region 2 FACs above the polar region in the Northern Hemisphere (Iijima and Potemra, 1976). We intend to improve the curlometer technique to calculate the current accurately for confirmation and investigation of the distributions of the region 2 FACs at the altitude of $4-5 R_{\mathrm{E}}$ in the future.

At large separations, all four spacecraft observed similar signatures one by one with few differences other than those due to convection of the FACs in time. The large separations and the abnormal tetrahedral configuration of the Cluster spacecraft array may reduce the validity of the 4-spacecraft technique. Nevertheless, the similar bipolar signatures observed one-by-one by the 4 Cluster spacecraft also suggest that the region 2 FACs are very stable and are almost stationary during the Cluster array crossing, which indicates 
the timing analysis should work well under these conditions. For example, the results from timing analysis confirm the FACs referred to in Sect. 4.2 for the events on 12 February 2007, when the average large separation between the 4 Cluster spacecraft is about $1600 \mathrm{~km}$. The deviations in the normal direction in this case also show a positive to negative bipolar signature at the pre-perigee crossing (see the left panel of the right part of Fig. 7a), and a less common negative to positive bipolar signature at the post-perigee crossing (see the right panel of the right part of Fig. 7a), which also indicate the Cluster spacecraft array might be crossing one or two poleward FACs in the pre-midnight sector. We find that the variations in the average $\boldsymbol{B}_{\mathrm{EO}-\mathrm{Navg}}$ are much larger than those in the average $d B_{\text {Navg }}$. This might be because T01 overestimates these FACs during quiet IMF conditions. Similar features can also be found in the residuals for the later years, resulting from the quiet external conditions during the years near solar minimum.

\section{Conclusion}

An investigation comparing eight years of magnetic field data from the 4 Cluster spacecraft with Tsyganenko 1989 (T89), 1996 (T96), and 2001 (T01) field models, while Cluster passes through, or adjacent to, the equatorial ring current, has been carried out which significantly extends that of Woodfield et al. (2007), both with different orbit locations and model versions. There are some differences in the comparisons of the data with the different models and with different spacecraft, and as a result of the changing magnetospheric location (due to the progressive dropping southward of the Cluster orbit). During the Cluster ring current crossing (through perigee, at 4-5 $R_{\mathrm{E}}$ ), the T01 and T89 models underestimate the ring current, while T96 overestimates it. This study confirms that the deviations between the data and the model take two forms: a sharp, bipolar signature and welldefined trends over a larger spatial region; these residuals can reach $\sim 70 \mathrm{nT}$ near perigee. We have shown here that these deviations are much weaker during the years close to the solar minimum, when geomagnetic activity is weaker. The sharp bipolar signatures are suggested to be Cluster crossings of the region 2 FACs or low-altitude cusp FACs, depending on dayside or nightside orientation. For Cluster region 2 crossings, only T96 and T01 include the region 2 FACs in the model structure and T01 performs better. Overall, the residuals from T01 are much smaller than from the other models for all of the magnetic field components, indicating that this model achieves the best fit to the data.

The four Cluster spacecraft observe nearly the same signatures at the small separations characteristic of the early years of the mission, but do sample different signatures at the large separations during the later years, allowing comparisons to be made during similar external conditions. Using the fourspacecraft technique, we find that the region 2 FACs, with a size of $\sim 0.17-0.54 R_{\mathrm{E}}$, and cusp FACs, with a size of $\sim 0.06$ $0.12 R_{\mathrm{E}}$, are very stable and are almost stationary during the Cluster array crossings.

In future, we hope to better map the magnetospheric response in this region generally, and to study the quiet and storm time ionospheric current systems linked via the region 2 FAC.

Acknowledgements. The authors wish to express their gratitude to the UK research council NERC for funding this work through the GEOSPACE consortium, grant number NER/0/S/2003/00675. We thank the Cluster FGM Operations Team and Cluster FGM PI, E. A. Lucek for the magnetic field data, and N. A. Tsyganenko for his models. We acknowledge the NASA OMNIWeb site to supply us the solar wind and IMF data and the World Data Center for Geomagnetism to supply the SYM-H magnetic activity index.

Topical Editor R. Nakamura thanks two anonymous referees for their help in evaluating this paper.

\section{References}

Balogh, A., Carr, C. M., Acuña, M. H., Dunlop, M. W., Beek, T. J., Brown, P., Fornacon, K.-H., Georgescu, E., Glassmeier, K.H., Harris, J., Musmann, G., Oddy, T., and Schwingenschuh, K.: The Cluster Magnetic Field Investigation: overview of in-flight performance and initial results, Ann. Geophys., 19, 1207-1217, 2001, http://www.ann-geophys.net/19/1207/2001/.

Bryant, D. A., Krimigis, S. M., and Haerendel, G.: Outline of the Active Magnetospheric Particle Tracer Explorers (AMPTE) Mission, IEEE Trans. Geosci. Remote Sensing, GE-23(3), 177-181, 1985.

Dunlop, M. W., Balogh, A., Glassmeier, K.-H., and Robert, P.: Four-point Cluster application of magnetic field analysis tools: The Curlometer, J. Geophys. Res., 107, 1384-1387, 2002.

Escoubet, C. P., Fehringer, M., and Goldstein, M.: The Cluster mission, Ann. Geophys., 19, 1197-1200, 2001, http://www.ann-geophys.net/19/1197/2001/.

Iijima, T. and Potemra, T. A.: The Amplitude Distribution of FieldAligned Currents at Northern High Latitudes Observed by Triad, J. Geophys. Res., 81, 2165-2174, 1976.

Maus, S., Macmillan, S., Chernova, T., Choi, S., Dater, D., Golovkov, V., Lesur, V., Lowes, F., Luhr, H., Mai, W., McLean, S., Olsen, N., Rother, M., Sabaka, T., Thomson, A., and Zvereva, T.: The 10th-Generation International Geomagnetic Reference Field, Geophys. J. Int., 161, 561-565, 2005a.

Maus, S., Macmillan, S., Chernova, T., Choi, S., Dater, D., Golovkov, V., Lesur, V., F. Lowes, Luhr, H., Mai, W., McLean, S., Olsen, N., Rother, M., Sabaka, T., Thomson, A., and Zvereva, T.: Short communication: The 10th generation international geomagnetic reference field, Phys. Earth Planet. In., 151, 320-322, 2005 b.

Shue, J. H., Song, P., Russell, C. T., Steinberg, J. T., Chao, J. K., Zastenker, G., Vaisberg, O. L., Kokubun, S., Singer, H. J., Detman, T. R., and Kawano, H.: Magnetopause location under extreme solar wind conditions, J. Geophys. Res., 103(A8), 17691-17700, 1998. 
Sugiura, M. and Kamei, T.: Equatorial DST index 1957-1986, in: IAGA Bull. 40, edited by: Bethelier, A. and Menvielle, M., Int. Serv. of Geomagn. Indices Publ. Off., Saint Maur, France, 1991. Sugiura, M., Ledley, B. G., Skillman, T. L., and Heppner, J. P.: Magnetospheric-Field Distortions Observed by Ogo 3 and 5, J. Geophys. Res., 76(31), 7552-7565, 1971.

Tsyganenko, N. A.: A model of the near magnetosphere with a dawn-dusk asymmetry 1. Mathematical structure, J. Geophys. Res., 107, 17(A8), 1179, doi:10.1029/2001JA000219, 2002a.

Tsyganenko, N. A.: A model of the near magnetosphere with a dawn-dusk asymmetry 2. Parameterization and fitting to observations, J. Geophys. Res., 107, 17(A8), 1176, doi:10.1029/2001JA000220, 2002b.

Tsyganenko, N. A.: A magnetospheric magnetic field model with a warped tail current sheet, Planet. Space Sci., 37, 5-20, 1989.

Tsyganenko, N. A. and Stern, D. P.: Modeling the global magnetic field of the large-scale Birkeland current systems, J. Geophys. Res., 101, 27187-127198, 1996.

Tsyganenko, N. A. and Sitnov, M. I.: Modeling the dynamics of the inner magnetosphere during strong geomagnetic storms, J. Geophys. Res., 110, A03208, doi:10.1029/2004JA010798, 2005.
Tsyganenko, N. A. and Russell, C. T.: Magnetic signatures of the distant polar cusps: Observations by Polar and quantitative modeling, J. Geophys. Res., 104(A11), 24939-24955, 1999.

Tsyganenko, N. A.: Magnetic field and electric currents in the vicinity of polar cusps as inferred from Polar and Cluster data, Ann. Geophys., 27, 1573-1582, 2009, http://www.ann-geophys.net/27/1573/2009/.

Vallat, C., Dandouras, I., Dunlop, M., Balogh, A., Lucek, E., Parks, G. K., Wilber, M., Roelof, E. C., Chanteur, G., and Rème, H.: First current density measurements in the ring current region using simultaneous multi-spacecraft CLUSTER-FGM data, Ann. Geophys., 23, 1849-1865, 2005, http://www.ann-geophys.net/23/1849/2005/.

Wanliss, J. A. and Showalter, K. M.: High-resolution global storm index: Dst versus SYM-H, J. Geophys. Res., 111, A02202, doi:10.1029/2005JA011034, 2006.

Woodfield, E. E., Dunlop, M. W., Holme, R., Davies, J. A., and Hapgood, M. A.: A comparison of Cluster magnetic data with the Tsyganenko 2001 model, J. Geophys. Res., 112, A06248, doi:10.1029/2006JA012217, 2007. 